\title{
Analysis of Acoustic Emission Propagation in Metal-to-Metal Adhesively Bonded Joints
}

\author{
Alasdair Crawford ${ }^{1} \cdot$ Mohamad Ghazi Droubi $^{1}$ (D) Nadimul Haque Faisal ${ }^{1}$
}

Received: 7 October 2017 / Accepted: 20 April 2018 / Published online: 4 May 2018

(c) The Author(s) 2018

\begin{abstract}
Acoustic emission (AE) monitoring shows promise as one of the most effective methods for condition monitoring of adhesively-bonded joints. Previous research has demonstrated its ability to detect, locate and classify adhesive joint failure, though in these studies little attention appears to have been paid to the differences in AE wave propagation through the bonded and un-bonded sections of the specimens tested, or to the effects of the wave modes excited or the propagation distances. This paper details an experimental study conducted on large aluminium sheet specimens to identify the effects of the presence of an adhesive layer on AE wave propagation. Three specimens are considered; a single aluminium sheet, two aluminium sheets placed together without adhesive, and an adhesively-bonded specimen. A pencil lead break (PLB) is used as a simulated AE source, and is applied to the three specimens at varying propagation distances and orientations. The acquired signals are processed using wavelet-transforms to explore time-frequency features, and compared with modified group-velocity curves based on the Rayleigh-Lamb equations to allow identification of wave-modes and edge-reflections. The effects of propagation distance and source orientation are investigated while comparison is made between the three specimens. It is concluded that while the wave propagation modes can be approximated as being constant throughout all three specimens, there is a significant change in the received waveforms due to the attenuation of high-frequency components exhibited by the bonded specimen. These findings may be utilised to provide a deeper understanding of acquired AE data, improving the current abilities to identify, locate and characterise damage mechanisms occurring within adhesive joints, ultimately improving safety in the use of adhesive bonding for critical applications.
\end{abstract}

Keywords Adhesive joints - Acoustic emission - Wave propagation · Wavelet transform · Signal processing · Pencil lead break (PLB) test

\section{Notations}

$\mathrm{A}_{0} \quad$ Fundamental anti-symmetric Lamb mode

$c_{l} \quad$ Longitudinal wave velocity

$c_{t} \quad$ Shear wave velocity

$c_{p} \quad$ Phase velocity

$h \quad$ Half the sheet thickness

$g \quad$ Group-velocity

$k \quad$ Wave number

$\mathrm{S}_{0} \quad$ Fundamental symmetric Lamb mode

Mohamad Ghazi Droubi

m.g.droubi@rgu.ac.uk

Nadimul Haque Faisal

n.h.faisal@rgu.ac.uk $\omega \quad$ Angular frequency

AE Acoustic Emission

ASTM American Society for Testing and Materials

CWT Continuous wavelet transforms

FEM Finite element method

FFT Fast fourier transform

kHz: Kilo hertz

$\mathrm{MHz} \quad$ Mega hertz

NI National instruments

PLB Pencil lead break

PSD Power spectral density

PTFE Polytetrafluoroethylene

STFT Short-time Fourier transform

WT Wavelet transform

2D-FFT Two-dimensional fast Fourier transform

1 School of Engineering, Robert Gordon University, Garthdee Road, Aberdeen AB10 7GJ, UK 


\section{Introduction}

Many techniques to assess adhesive bond quality have been developed, with varying levels of success and differing merits in terms of accuracy and practicality. Various ultrasonic techniques, such as through-transmission, pulse-echo and pitch-and-catch systems are widely used throughout industry. While they are extremely effective in certain situations, they can be limited by aspects such as; requiring access to both sides of the bond (for through-transmission), the limited depth that can be inspected by single-sided approaches, the necessity for sensor coupling (usually achieved by waterjet or immersion bath), and the inability to detect certain defects such as zero-volume disbonds. These techniques are also reliant on scanning of the entire area being inspected, an extremely time-consuming process for large areas, with areas of several square metres potentially taking over an hour to scan, depending on the desired resolution [1]. Techniques such as radiography and infrared-thermography can inspect larger areas much faster, however radiography is largely insensitive to the presence of adhesive unless it is combined with a metallic filler, as the density of the adherends is generally much higher than that of the adhesive [2]. While infrared thermography provides a similar sensitivity to near-surface defects as ultrasonic pulse-echo techniques, it is less sensitive to deeper defects and is generally unsuitable for inspection of both particularly thin layers and specimens made of highly conductive materials, such as metals [1]. A variety of other techniques including impedance, and sonic and ultrasonic vibration based methods are also available and have their own advantages, the majority, however, are still restricted by the time-consuming requirement of scanning of the bond area. One technique which avoids this issue is acoustic emission.

Acoustic emission (AE) has proven promising for condition monitoring of large structures and has been shown to be effective in detecting failure of adhesive joints [3-9]. AE is a passive technique based on the detection of transient elastic waves released by the sudden redistribution of stress resulting from processes such as crack initiation and growth. It is capable of detecting multiple damage mechanisms (e.g. adhesive failure/interfacial debonding [3,6,9], cohesive failure/adhesive cracking $[9,10]$, adherend matrix cracking [11], fibre breakage [3,11], sandwich core failure [11]) and is not limited by a minimum detectable defect size.

The possibility of utilising AE for the condition monitoring of adhesive bonds has been explored by several researchers. Several studies, (e.g. Droubi et al. [6,7]; Senthil et al. [10]) have recognised the correspondence between the initiation of AE activity and changes in the load-displacement curves resulting from the onset of adhesive failure, during mechanical testing of various bonded specimens. Droubi et al. [6] investigated the mode I and II failure of bonded metal- to-metal and metal-to-composite specimens through use of $\mathrm{AE}$ instrumented double cantilever beam and three-point end-notch-flexure tests. Both a ductile and a brittle adhesive were investigated with varying levels of bond quality, introduced by use of Polytetrafluoroethylene (PTFE) spray to reduce the effective bond area. As well as noting correspondence between AE activity and features in the load-curves it was also recognised, during both calibration tests using a pencil lead break and during debonding, that there was an increase in both $\mathrm{AE}$ amplitude and in the proportion of higher frequency spectral content as the source moved closer to the sensor. Analysis was conducted by Fast Fourier Transform (FFT) and by energy content after bandpass filtering into low, medium and high frequency ranges, and therefore considered the entire hit, including the multiple edge reflections likely in small specimens. Bak and Kalaichelvan [3] have differentiated between failure mechanisms of fibre tear, light fibre tear and adhesive failure by analysis of the peak frequencies of each hit during lap-shear testing of glass fibre composite and pure resin single- and doublelap-joint specimens. Comparison of the AE peak frequencies with scanning electron microscope images allowed identification of correspondence between the failure mechanisms and peak frequencies. The use of a second sensor also allowed for source location of each hit for further validation of the failure mechanisms. While this method appears successful in the small specimens tested ( $25.4 \mathrm{~mm}$ square bond area), where the source-sensor distance experiences minimal variation, the results presented by Droubi et al. [6] indicate that changes in source-sensor separation may lead to changes in spectral content, and thus in the failure-mechanism recognised. For application of this method to larger bond areas the effects of propagation distance may need to be accounted for to ensure reliability of results. Galy et al. [9] have also successfully differentiated between failure-mechanisms of aluminium and epoxy lap-shear specimens. By use of the kmeans clustering method, with inputs of temporal features including amplitude, energy, duration and rise time, it was possible to attribute $\mathrm{AE}$ hits to either debonding between the adhesive and adherends, or cracking of the adhesive. As with most of the works relating to $\mathrm{AE}$ testing of adhesive bonds, the bonded area of the specimens used was of a standard size for a lap-shear test $(25 \times 12.5 \mathrm{~mm})$, resulting in minimal variation in propagation distance. As in Bak and Kalaichelvan [3], the application of this method to larger specimens, in which propagation distances will vary more significantly, should be approached cautiously as the dispersion of $\mathrm{AE}$ waves with increasing propagation distance may lead to reduced amplitude and energy, and variation in duration and rise time, while edge reflections will also play a more complex role in affecting these factors dependent on the geometry. As AE has been shown to be capable of not only detecting damage in adhesive bonds but also of locating 
and categorising it, it is of significant interest to investigate the effects of AE propagation through bonded specimens, as this is a factor which will be critical to the ability to successfully apply the existing experimental methods to large scale applications.

In the case of thin sheets, which are of primary interest for adhesive bonding, AE energy propagates as "Lamb" or "Plate" waves, with multiple wave modes propagating simultaneously and the group-velocity of each of these modes being frequency dependent. Due to this, dispersion of the waves occurs over distance, causing significant changes to the typical AE features recorded such as peak-amplitude, rise-time and duration. The identification of the correct wave modes and corresponding group velocities is also vital for the accuracy of any time-of-arrival based source location methods. As previously stated however, previous AE studies of adhesive joints have paid little attention to the types of waves generated by the events within the adhesive, or to how these waves have propagated to the sensor. Other researchers have, however, studied the propagation of $\mathrm{AE}$ waves in thin aluminium plates [12-14]. Hamstad et al. [14] have used AE (generated from a pencil lead break source) applied to the edge of a plate to excite low order Lamb waves where acquired $\mathrm{AE}$ signals were recorded using broadband transducers and then converted to the time-frequency domain through use of the wavelet transform technique. Comparison of the high-energy regions of the WT coefficient plots with modified group-velocity curves allowed clear identification of the wave modes propagating in the specimen. This technique has also been applied to the more complex geometry of a section of rail track by Zhang et al. [15] to assess the effects of propagation distance and source depth. Moreover, the propagation of Lamb waves within thin adhesively-bonded aluminium specimens has previously been studied by Heller et al. [16], using laser ultrasonic and a 2D-FFT processing technique to generate Lamb wave dispersion curves. Comparison of single aluminium sheets, bonded specimens and aged bonded specimens revealed that the same wave modes existed in all specimens and corresponded to the dispersion curves of a single aluminium sheet, although the addition and subsequent degradation of an adhesive layer resulted in the disappearance of high frequency content corresponding to higher order wave modes.

The aim of this study is to investigate the AE wave propagation in a single layer, an un-bonded double-layer and an adhesively-bonded aluminium joint, with both in-plane and out-of-plane sources, to provide more insight into the propagation of AE waves excited by defect propagation in bonded joints. Edge reflections have also been included in this investigation rather than being isolated and removed, as these make up a significant part of AE hits which are typically analysed.

\section{Experimental Setup}

\subsection{Sample Preparation}

The essential experimental approach was to carry out a systematic investigation of $\mathrm{AE}$ wave propagation in large aluminium sheets, that investigation spanning over a single sheet, two identical sheets placed on top of each other without adhesive (un-bonded double-layer), and an adhesivelybonded specimen. 1050 A H14 aluminium substrate sheets of average thickness $1 \mathrm{~mm}$ (Grampian Steel Services, UK) were cut into sections of $500 \mathrm{~mm} \times 500 \mathrm{~mm}$. For the adhesivelybonded specimen, the adherends were first abraded by hand with $\mathrm{P} 400$ grade abrasive paper then rinsed with acetone and cleaned using LOCTITE® SF $7063^{\mathrm{TM}}$. The mean surface roughness $\left(R_{a}\right)$ before bonding was $1.18 \mu \mathrm{m}$. This was measured across 20 different areas on the adherends using a Taylor Hobson Surtronic 3+ surface roughness tester. Following the process of surface preparation, the LOCTITE® EA 9461 adhesive (a typical thixotropic two-part epoxy, chosen for its ease of application and room-temperature curing) was applied uniformly to the entire surface of a single sheet using a clean aluminium spreading stick. The opposite sheet was carefully and accurately placed on top and a flat plate with weights, totalling $180 \mathrm{~N}$, was placed on top of the specimen to create a uniform load. The adhesive thickness was not directly controlled during this process. The specimen was left to cure for five days to achieve full strength before being handled. Average room temperature and relative humidity during curing were approximately $19^{\circ} \mathrm{C}$ and $41 \%$, respectively. The mean cured adhesive thickness (calculated from 40 thickness measurements taken around the edges of the specimen using a micrometer) is $0.2 \mathrm{~mm}$, with a standard deviation of $0.11 \mathrm{~mm}$.

\subsection{Acoustic Emission Instrumentation}

The experimental setup shown in Fig. 1 consists of the test specimen and AE measurement system. A Micro-80D differential AE sensor (Physical Acoustics Ltd, UK) was used throughout the investigation. This sensor type provides a relatively flat frequency-response over a range of approximately $175-900 \mathrm{kHz}$, with a peak at around $320 \mathrm{kHz}$. As with any acoustic emission system, the frequency-response of the sensor will be reflected in the recorded signal, and thus the use of a different sensor may provide results with significantly different spectral content. The same sensor was therefore used across all specimens, ensuring variation in results arises from differences in the specimens and not the sensors. Silicone grease was applied to the sensor (to avoid any air gap) 
Fig. 1 Schematic layout of the experimental setup including: test specimen, AE sensor (assembled at the centre of the specimen using aluminium tape), pre-amplifier, signal-conditioning-unit, shielded connector-block and desktop PC with data acquisition card

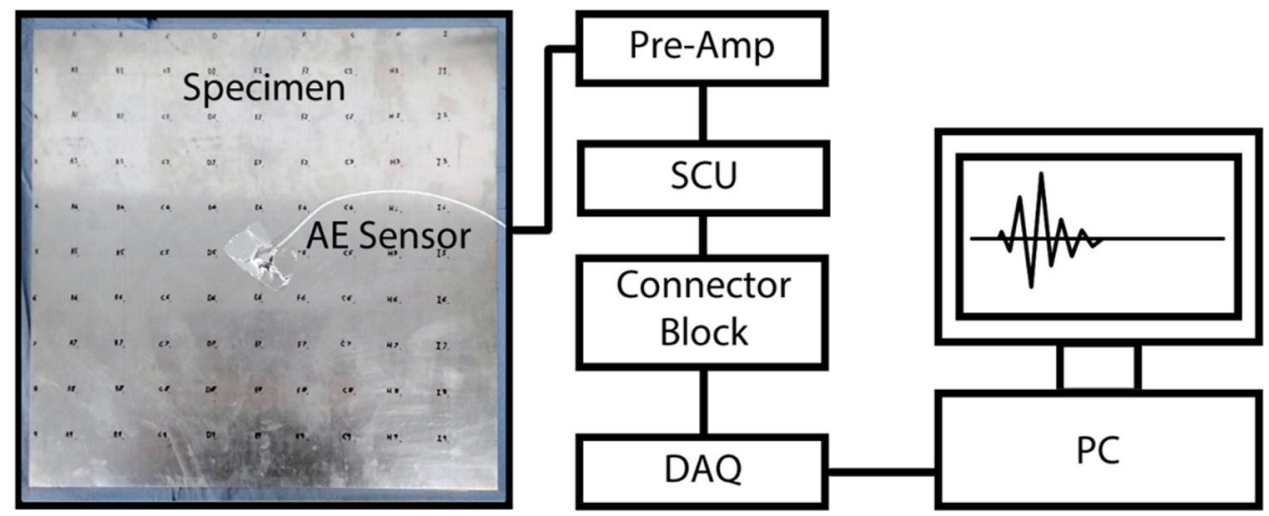

before attaching to the specimen surface using aluminium tape. Aluminium tape was chosen over the more commonly used magnetic clamps due to the use of non-magnetic aluminium adherends.

As shown in Fig. 1, the AE sensor was connected to a preamplifier that was utilised to amplify the acquired signals gain and could be varied $(20 / 40 / 60 \mathrm{~dB})$. The pre-amplifier also featured an integrated $20 \mathrm{kHz}$ high-pass filter. The pre-amplifier was connected to an in-house-built 4-channel signal-conditioning-unit (SCU) that was coupled with a gainprogrammer to provide a $28 \mathrm{~V}$ power-supply, coupled with adjustable gain control. The SCU transmits the adjusted signal to a National Instruments (NI) BNC-2120 shielded connector-block to complete the systems signal transmission to the data acquisition card (DAQ). The signals were interpreted through a computer using a $10 \mathrm{MS} / \mathrm{s}$ NI PCI-6115 DAQ to obtain the raw signal data and convert it to a binary file within the LabVIEW software for further analysis using MATLAB. A pencil lead break test is a well-established procedure for generating simulated AE sources (Hsu-Nielsen source [17]). Therefore, a commercial mechanical pencil with an in-house machined guide-ring was used to generate simulated AE sources by breaking a $0.5 \mathrm{~mm}$ diameter and 2-3 mm length $2 \mathrm{H}$ pencil lead, as recommended by ASTM standards (E976-99) [17]. A Hsu-Nielsen source provides a signal with a broad frequency spectrum, with significant content in the bandwidths previously demonstrated to be associated with adhesive failure $[3,6,11]$. This, combined with its good repeatability, makes it an appropriate choice of source for these experiments. AE signal acquisition for the mechanical testing of the specimens were set at (SCU gain: $12 \mathrm{~dB}$, pre-amp gain: $40 \mathrm{~dB}$, sampling frequency: $2 \mathrm{MHz}$, signal acquisition time: $0.025 \mathrm{~s}$ ). The sampling frequency was chosen to be more than twice the maximum frequency being investigated, thus exceeding the requirements of the Nyquist Sampling Theorem and avoiding potential aliasing of the signal [18].

\subsection{Pencil Lead Break (PLB) Test Procedure}

For all experiments carried out in this study, the AE sensor was positioned on the face of the specimen in its centre, and the waveforms propagating were recorded with the simulated source placed along the centreline at 50,100, 150 and 200 $\mathrm{mm}$ away from the specimen centre as shown in Fig. 2. To assess the effects of source orientation, the simulated $\mathrm{AE}$ source was also applied to the edges of the specimens at 250 $\mathrm{mm}$ from the sensor, as shown in Fig. 2. The source applied to the edge of the specimens was applied at the mid-depth of the specimen in the case of the single sheet. On the un-bonded double-layer specimen, it was applied to the mid-depth of the upper sheet. For the bonded specimen, the source was applied at the mid-depth of the upper adherend and on the adhesive layer at the mid-depth of the entire specimen. Tests were initially carried out with the source on the same face as the sensor, the tests were then later repeated with the source applied to the same locations but on the opposite sides of the specimens from the sensor. To ensure repeatability, five pencil lead breaks were recorded at each of the positions as per Fig. 2.

\section{Acoustic Emission Signal Processing Method}

\subsection{Wavelet Transforms}

In the analysis of thin sheets, it is particularly important to be able to conduct analysis in both time and frequency domains. The dispersive nature of Lamb or Plate waves results in the propagation of multiple wave modes with different frequency components travelling at different velocities. Combined with the effects of edge reflections this makes in-depth analysis in either time or frequency domain alone extremely challenging, particularly where components may overlap and edge 


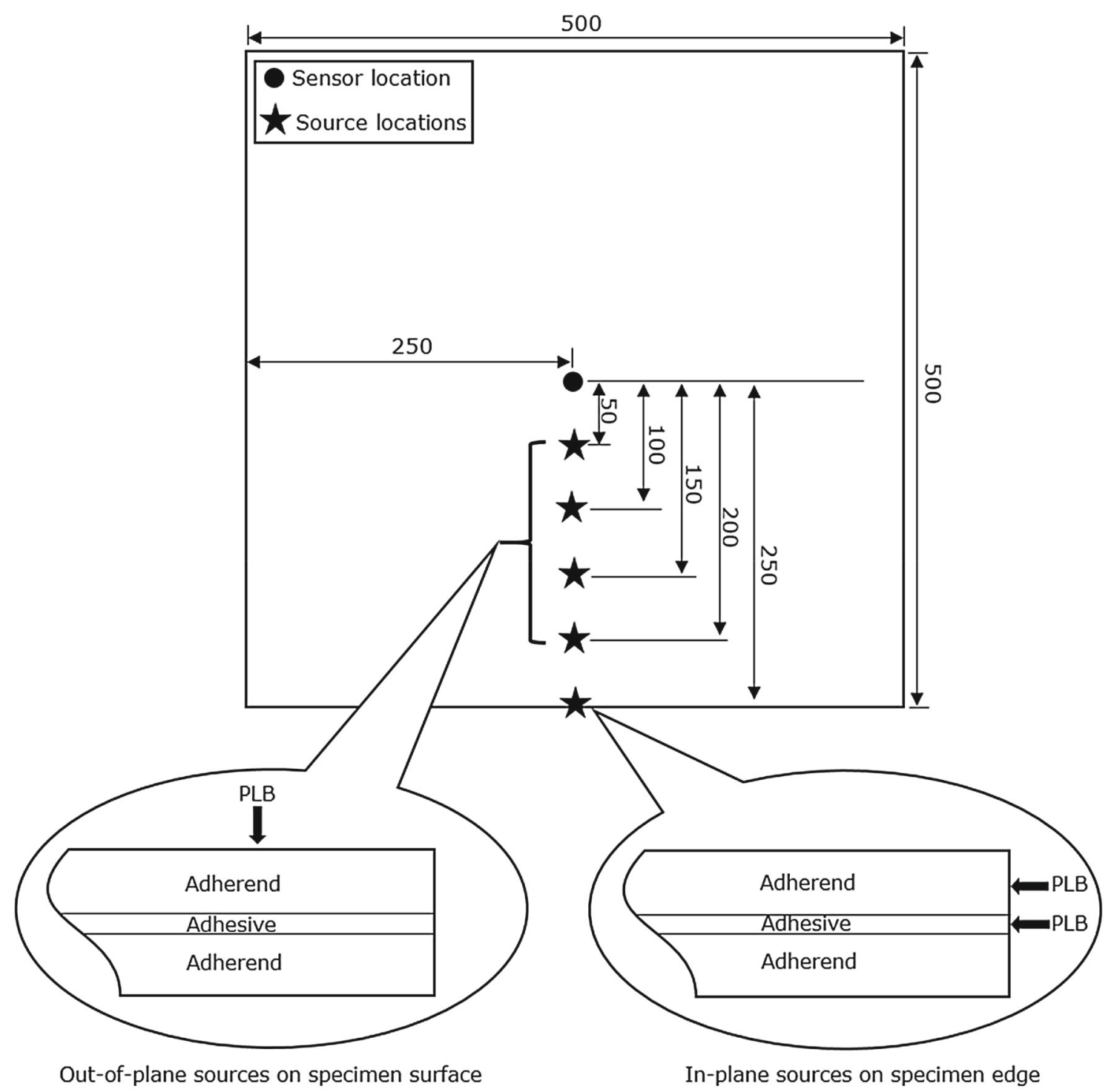

Fig. 2 Source and sensor locations used for all specimens. PLB applied on sheet surface to create an out-of-plane source at 50,100,150 and $200 \mathrm{~mm}$ from the sensor. PLB applied to edge of the sheet (and edge of

the adhesive for the adhesively-bonded specimen) to create an in-plane source $250 \mathrm{~mm}$ from the sensor. All dimensions in millimetres

reflections may occur prior to the arrival of slower travelling components of the initial wave.

To allow analysis of the recorded AE waveforms in both time and frequency domains the wavelet transform (WT) has been utilised. The continuous wavelet transforms (CWT) is advantageous over other time-frequency representations, such as Short-Time Fourier Transform (STFT), as it is not restricted to a fixed window length across all frequencies. Using a longer window at lower frequencies and a shorter window at high frequencies ensures good frequency resolution at low-frequency and good time resolution at highfrequency, features that are lost by use of a fixed window length. A full description of the wavelet transform method

applied to acoustic emission signals can be found in the work of Suzuki et al. [19]. In this study wavelet transforms were implemented using open access AGU-Vallen Wavelet software (ver. R2015.0430.6), with further analysis of the WT being carried out in MATLAB (ver. 7.9.0.529 (R2009b)). The wavelet software utilises a Gabor-type wavelet as this is known to provide the best combination of time and frequency resolution of any available wavelet as the product of its standard deviations in both time and frequency domains are minimised. A relatively small wavelet-size setting of 50 samples was used, prioritising fidelity of the time-domain over the smoothness which would be achieved by use of a larger wavelet-size setting. 
Fig. 3 Group-velocity curves for a $1 \mathrm{~mm}$ aluminium sheet. $A_{0-4}$ represent the four lowest order anti-symmetric waves and $S_{0-4}$ represent the four lowest order symmetric waves

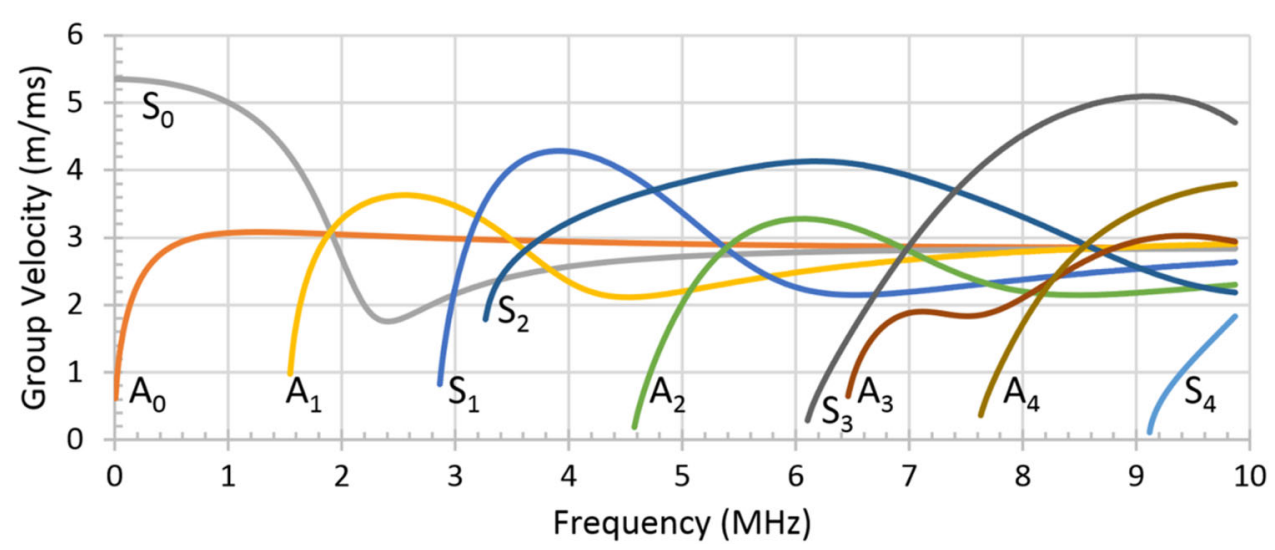

\subsection{Rayleigh-Lamb Equations}

To allow identification of the different Lamb wave modes and edge reflections present within the acquired wavelet transforms, the theoretical arrival times of these different features have been calculated from the group-velocity curves based on the Rayleigh-Lamb equations. While providing a full description of Lamb wave theory is out-with the scope of this paper, a good introduction to the topic can be found in $\mathrm{Su}$ and Ye [20]. The Rayleigh-Lamb equations are given as Eqs. (1) and (2) below [20]:

Symmetric modes: $\frac{\tan (q h)}{\tan (p h)}=-\frac{4 k^{2} p q}{\left(k^{2}-q^{2}\right)^{2}}$

Anti-symmetric modes: $\frac{\tan (q h)}{\tan (p h)}=-\frac{\left(k^{2}-q^{2}\right)^{2}}{4 k^{2} p q}$

where: $p^{2}=\frac{\omega^{2}}{c_{l}^{2}}-k^{2}, q^{2}=\frac{\omega^{2}}{c_{t}^{2}}-k^{2}$

$h$ is half the sheet thickness, $\omega$ is the angular frequency, $k$ is the wave number, $c_{l}$ is longitudinal wave velocity and $c_{t}$ is shear wave velocity. Phase velocity: $c_{p}=\omega / k$ and groupvelocity $g=d \omega / d k$ are then calculated. Based on these equations, group-velocity curves, representing the variation in wave propagation velocities with frequency, can be plotted. In this study, this process of generating group-velocity curves has been carried out using open-access Vallen Dispersion software (ver. R2015.0430.6), using the software's pre-set values of $6420 \mathrm{~m} / \mathrm{s}$ and $3040 \mathrm{~m} / \mathrm{s}$ for longitudinal and shear wave velocities in aluminium. These values were verified by an additional test, in which two AE sensors were placed at positions $200 \mathrm{~mm}$ apart, on the centreline of the single sheet, with the simulated source also applied on the centreline at a distance of $50 \mathrm{~mm}$ from the first sensor. The method of overlaying dispersion curves, modified by the appropriate propagation distance as described in the following paragraph, was then used to verify the velocities. The group-velocity curves for the five lowest order wave modes of a $1 \mathrm{~mm}$ thick aluminium sheet are shown below in Fig. 3 . For this thickness of specimen, only the zero-order symmetric $\left(\mathrm{S}_{0}\right)$ and anti-symmetric $\left(\mathrm{A}_{0}\right)$ modes exist at frequencies under $1 \mathrm{MHz}$, meaning that for frequencies generally considered in AE there will be only two wave modes propagating and therefore higher order modes can be neglected, simplifying any analysis. At these relatively low frequencies the group velocities of the two modes are significantly different; given suitable propagation distance, the arrivals of these modes should therefore appear well separated in the time domain.

To allow comparison of the group-velocity curves with the WT coefficient plots, the velocities at each frequency are converted to arrival times based on the known propagation distance for each test. This technique has previously been successfully demonstrated by Hamstad et al. [12-14], considering AE wave propagation from in-plane and outof-plane sources in a large aluminium plate specimens, as well as having been applied to analysis of the more complex geometry of a section of rail track by Zhang et al. [15]. As a pencil lead break source is being used in this study, the exact application time is unknown and cannot be used to align the theoretical dispersion curves with the recorded signal. The curves have therefore been aligned with the acquired time and time/frequency plots in MATLAB, by aligning the earliest arriving group-velocity component with the first threshold crossing of the recorded time domain signal.

\subsection{Edge Reflections}

Both fundamental symmetric $\left(\mathrm{S}_{0}\right)$ and anti-symmetric $\left(\mathrm{A}_{0}\right)$ mode Lamb waves are subject to multiple reflections from the edges of the specimens, and reflections will continue to occur until the waves have been fully attenuated. Due to the relatively large size of the specimens tested, reflections generally appear well separated from the initial waves in the time domain, allowing separation and identification of these 


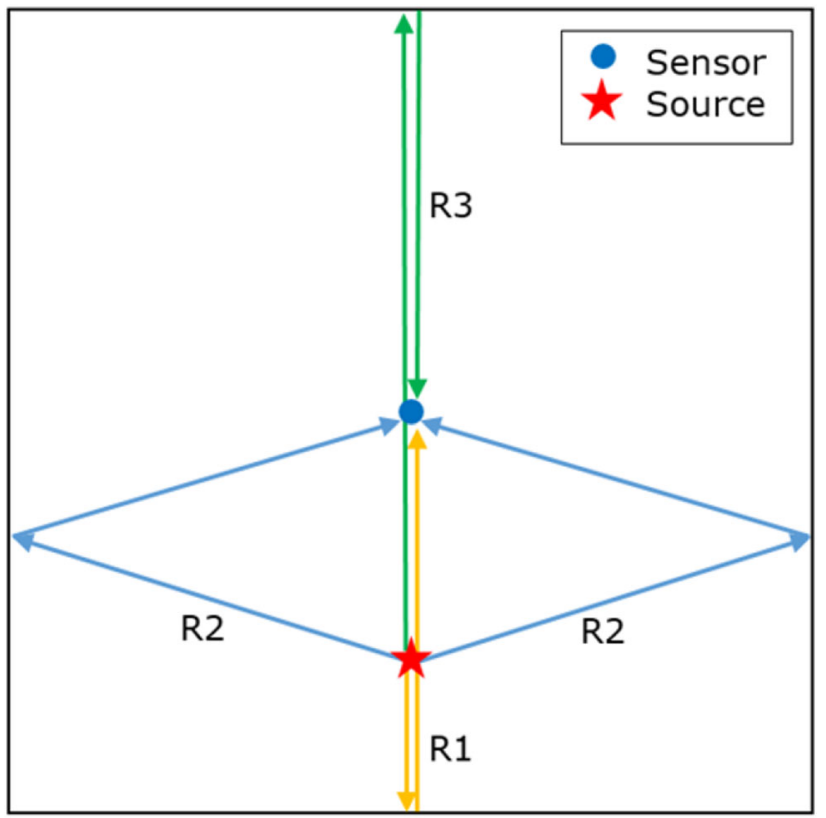

Fig. 4 Predicted wave propagation paths from source to sensor by means of edge reflections: (R1) Near-edge reflections, propagating from the source to the closest edge and returning to the sensor along the centreline. (R2) Side-edge reflections, propagating diagonally from the source to the left and right edges of the specimen and reflecting back to the sensor at a corresponding angle. (R3) Far-edge reflections, the reflection of the initial wave recorded, as it propagates from the source, past the sensor and reflects back along the centreline to the sensor from the far edge of the specimen

waves. Identification of the reflections and their propagation paths provides a clearer understanding of the features appearing in the WT coefficient plots. Group-velocity curves have been converted to arrival times based on propagation distance for the first three reflections, as was done for the initial $S_{0}$ and $\mathrm{A}_{0}$ waves. The propagation paths and distances used for the reflections, as shown in Fig. 4, are: the near-edge reflections (labelled R1 in following figures), from the source to the closest edge and back to the sensor all along the centreline of the specimen, the side-edge reflections (labelled R2 in following figures), from the source to the side edges of the specimens at an angle and then reflecting back to the sensor at the corresponding angle, and the far-edge reflections (labelled R3 in following figures), from the source to the furthest edge and back to the sensor all along the centreline of the specimen. Due to symmetry of the test setup, reflections from both side edges occur simultaneously. By overlaying the converted group-velocity curves on the WT plots, highenergy regions can be attributed to certain reflections. This is illustrated in Fig. 5. This method of identifying reflections has been successfully extended to identify more than three reflections, but only the first three are presented for the sake of brevity.

\subsection{Wavelet Transform Example}

Figure 5 illustrates an example of the techniques previously described. The lower window of the figure shows the original time domain signal recorded from the sensor. The upper window presents a contour plot of the wavelet transform coefficients. The red, high-energy, regions of the WT plot can be seen to correspond with high amplitude regions of the time domain signal, while the lower-energy regions correspond to lower amplitude regions. The group-velocity curves overlaid on the upper plot represent the theoretical arrival times of the initial zero order wave modes (shown in red), the reflections from the near edge (yellow), side edges (blue) and furthest edge (green). The alignment of the modified groupvelocity curves based on the first threshold crossing of the time domain signal is shown to be effective, as the curves are seen to align well with high-energy regions of the WT coefficient plot. In the time-frequency domain, and with the aid of the overlaid curves, it is possible to assess the contributions of each wave mode and each reflection to the overall signal in a way which is not possible from analysis in the time domain alone.

\section{Results and Discussion}

\subsection{Applicability of Group-Velocity Curves}

Figures 6, 7 and 8 show the WT coefficient plots and modified group-velocity curves for an out-of-plane source applied at propagation distances of 50,100, 150 and $200 \mathrm{~mm}$ on the single-sheet, un-bonded double-layer and bonded specimens. All specimen types exhibit the same basic features in the time-frequency domain: A low-amplitude peak with frequency content between 200 and $400 \mathrm{kHz}$ signals the arrival of the initial $\mathrm{S}_{0}$ wave, this is followed by the $\mathrm{A}_{0}$ wave, which contains three prominent regions. The first appears in the high frequency region above $400 \mathrm{kHz}$ and is of short duration. The second region corresponding to the $\mathrm{A}_{0}$ wave occurs in the $200-400 \mathrm{kHz}$ region, around the peak frequency of the sensor, and continues for a significantly longer duration than the first region. The third region occurs at low-frequency, under $100 \mathrm{kHz}$, and is of relatively long duration. These highenergy regions corresponding to both $\mathrm{S}_{0}$ and $\mathrm{A}_{0}$ waves recur throughout the signal as the waves are reflected by each of the edges. Increasing propagation distance leads to increasing the effects of dispersion, and thus also the separation between $A_{0}$ and $S_{0}$ waves. Moving the source further from the sensor is also seen to affect the arrival times of the reflections, as the propagation distance for a near-edge reflection is reduced, while the propagation distances for side-edge and far-edge reflections is increased. At a propagation distance of 
Fig. 5 Top: Example WT coefficient plot with overlaid group-velocity curves corresponding to initial $S_{0}$ and $A_{0}$ waves and their subsequent edge reflections. Bottom: Original time domain signal. Example shown for single-sheet specimen with out-of-plane source and $150 \mathrm{~mm}$ propagation distance
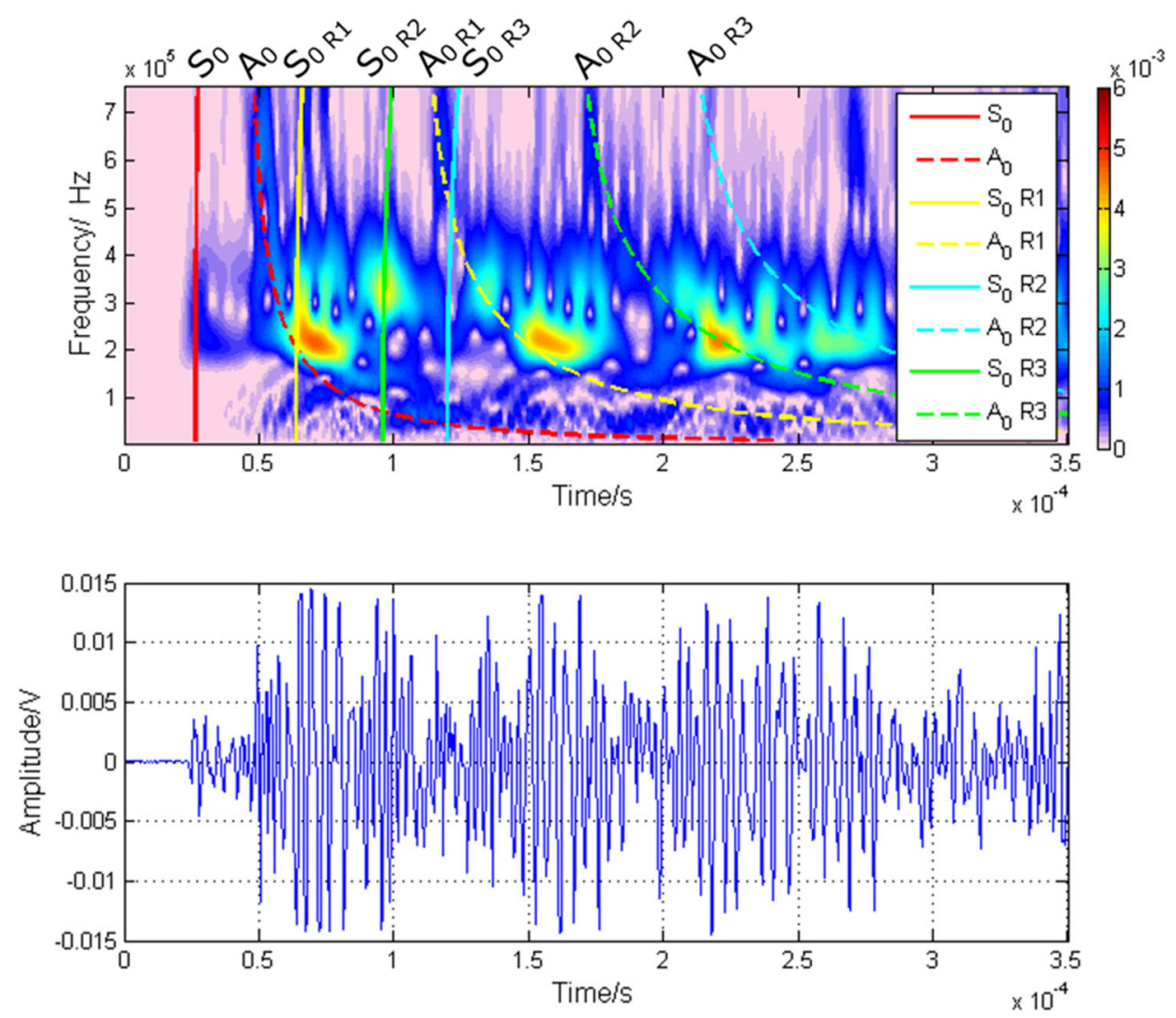

$200 \mathrm{~mm}$ the $\mathrm{S}_{0}$ reflection from the near-edge arrives before the $\mathrm{A}_{0}$ component of the initial wave.

It was found that all specimens tested show good correspondence between high-energy regions of the WT coefficient plots and the modified group-velocity curves generated for a single $1 \mathrm{~mm}$ sheet. In the case of the un-bonded doublelayer and bonded specimens, multiple dispersion curves based on different sheet-thicknesses, corresponding to their total thicknesses, were tested. These were however found not to fit as well as those for a $1 \mathrm{~mm}$ sheet. Over small propagation distances, where dispersion is minimal, there is little apparent difference between the curves, though at longer propagation distances the difference is apparent. Figure 9 shows the WT plot for the bonded specimen with a propagation distance of $200 \mathrm{~mm}$. Overlaid on this plot are the theoretical dispersion curves for a $1 \mathrm{~mm}$ sheet and a $2.2 \mathrm{~mm}$ sheet. The curves for a $1 \mathrm{~mm}$ sheet are seen to fit the WT plot well, passing through the peaks of the high energy regions corresponding to the $\mathrm{S}_{0}$ and $\mathrm{A}_{0}$ regions. The curves for a $2.2 \mathrm{~mm}$ sheet, however, do not pass through the peaks of the high energy regions. The $A_{0}$ curve occurs significantly earlier than the recorded features in the WT plot, this is particularly prominent at low frequency where dispersion is most significant.

The correspondence between high-energy regions of the WT coefficient plots and the modified group-velocity curves for a $1 \mathrm{~mm}$ aluminium sheet for all specimens implies that the presence of an additional layer, whether bonded or un-bonded, does not significantly affect the AE wave propagation in a sheet specimen in terms of wave velocities. The multi-layered specimens do not act as a single thicker specimen through which a single $\mathrm{S}_{0}$ and $\mathrm{A}_{0}$ waves would propagate, rather they act as multiple individual layers, with Lamb waves being excited individually in each layer. In the un-bonded specimen, this will occur as the layers are not actually connected; it is expected that due to the imperfect finish of the sheets there will be a small air-gap between the layers for most of the specimen's area. In the case of the adhesively-bonded specimen, the relatively low stiffness of the adhesive compared to the aluminium adherend permits the adherend to behave as a single sheet, with the presence of the adhesive layer having negligible effect on the wave behaviour. This finding mirrors those of Heller et al. [16], who concluded that the modes excited in bonded specimens are identical to the dispersion curves of a single plate, and fit well with the findings of Seifried et al. [21], whose analytical and FEM investigations based on the work of Heller et al. [16] lead to the conclusion that while additional wave modes are introduced by the presence of the adhesive and second adherend, only those close to the modes of the single adherend result in significant displacement of the surface of the adherends. 
Fig. 6 Example WT coefficient plots and modified

group-velocity curves for an out-of-plane source applied to the single aluminium sheet specimen with source-sensor propagation distances of a 50 $\mathrm{mm}, \mathbf{b} 100 \mathrm{~mm}, \mathbf{c} 150 \mathrm{~mm}$ and $\mathbf{d}$ $200 \mathrm{~mm}$

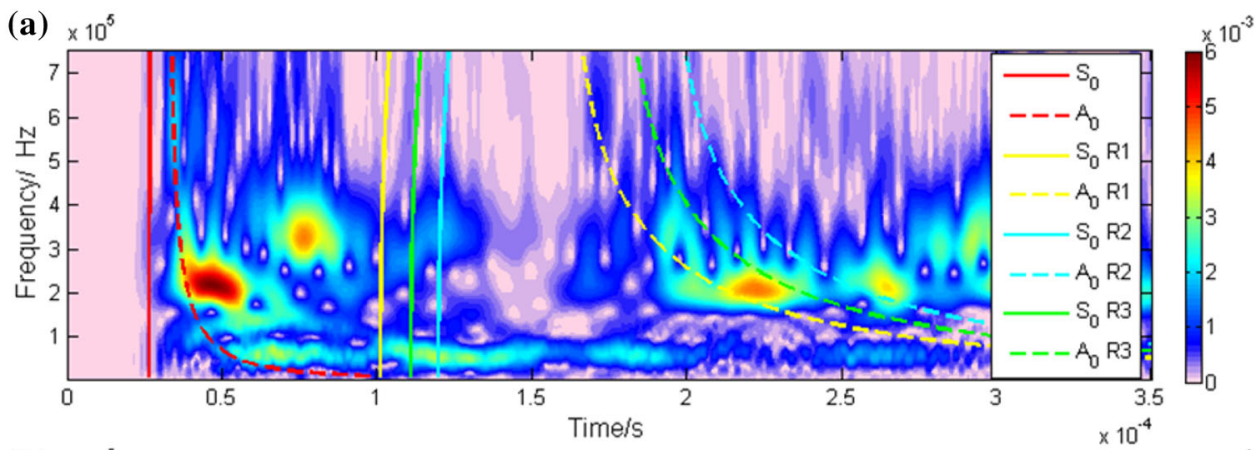

(b)
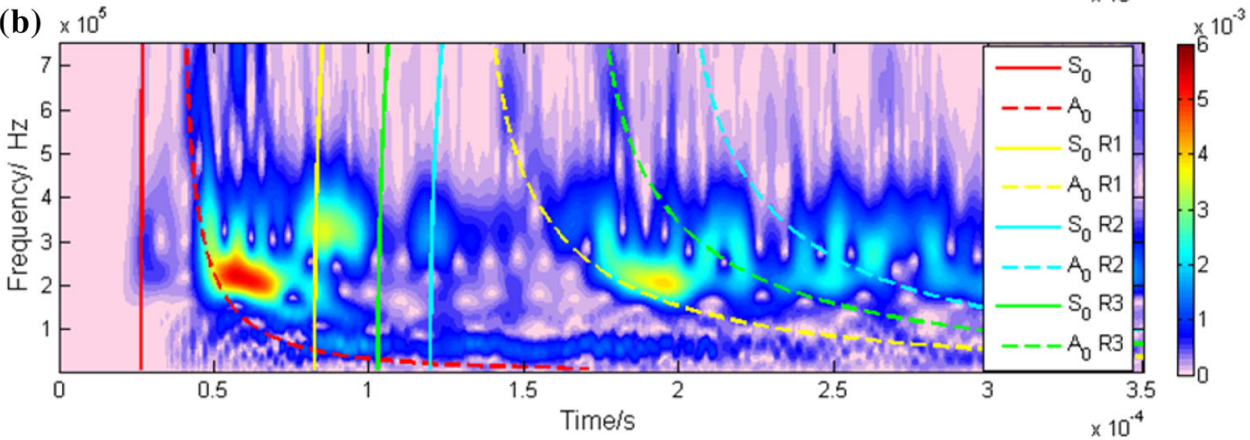

\section{(c)}

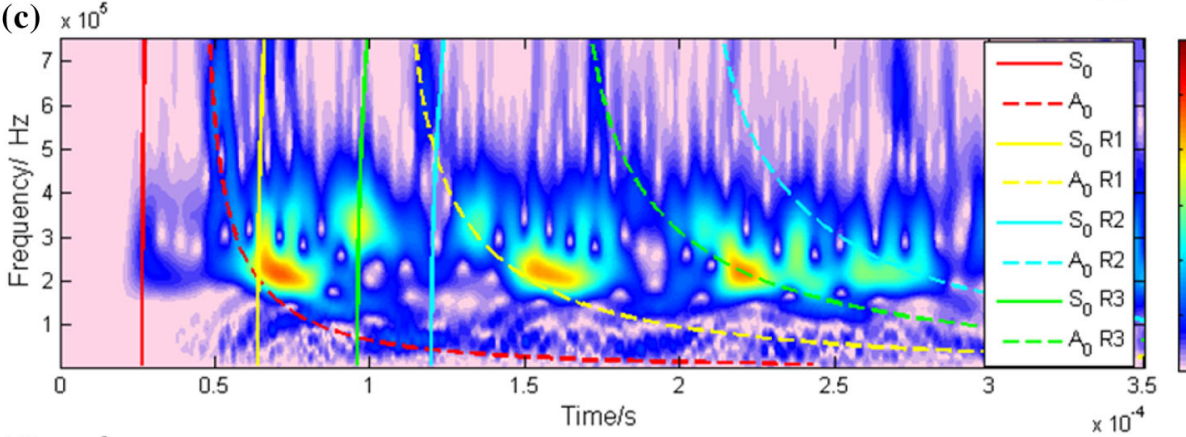

$\int_{-5}^{6^{6}} 0^{-3}$

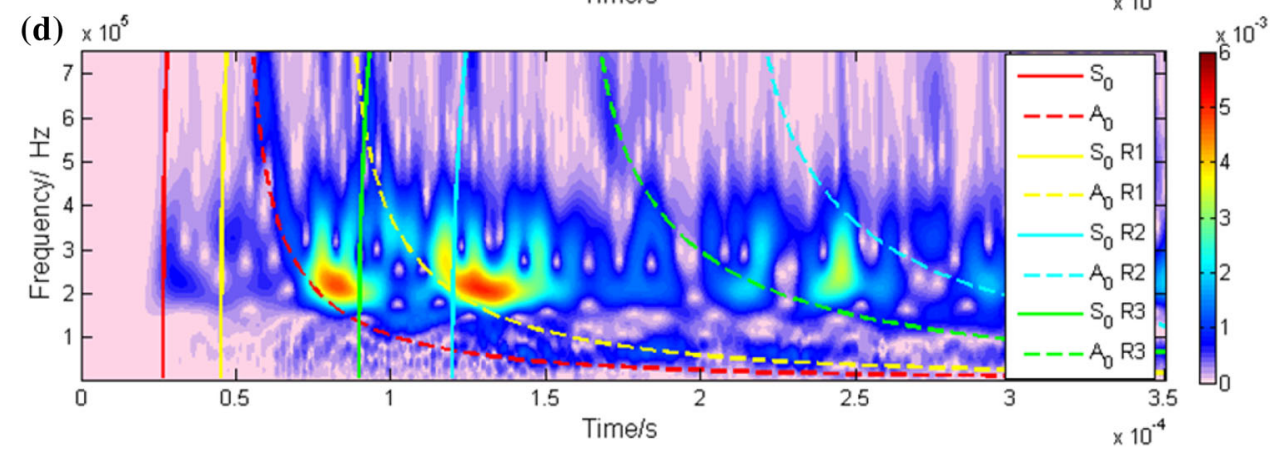

Regarding AE testing of bonded joints, this finding is particularly useful in the case of time-of-arrival based source location techniques as the theoretical dispersion curves for the adherend may be used to accurately identify the wave velocities to be used, regardless of bond status, bond-line thickness or presence of defects.

\subsection{Frequency Domain Analysis}

While all three specimens exhibit similar Lamb wave behaviour there are significant differences in the spectral con- tent of the recorded signals and in the changes to this content resulting from variation in propagation distance. Figure 10 shows power spectral density (PSD) plots, illustrating the frequency peaks for the entire signals recorded on the three specimens at the varying propagation distances. For ease of comparison, the plots are normalised by division of all PSD values by the peak value in each data set. While the recorded frequency content is largely determined by the frequency response of the sensor, which has a number of local peaks, the differences between specimens is significant; the single sheet exhibits content in frequency bands centred around approxi- 
Fig. 7 Example WT coefficient plots and modified group-velocity curves for an out-of-plane source applied to the un-bonded double-layer aluminium sheet specimen with source-sensor propagation distances of a $50 \mathrm{~mm}, \mathbf{b} 100$ $\mathrm{mm}, \mathbf{c} 150 \mathrm{~mm}$ and $\mathbf{d} 200 \mathrm{~mm}$

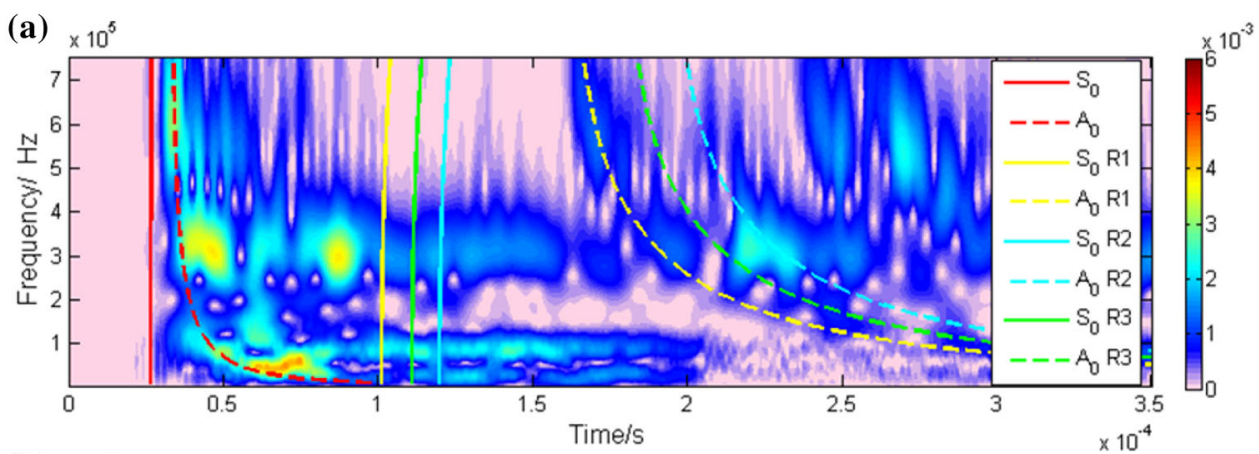

(b)

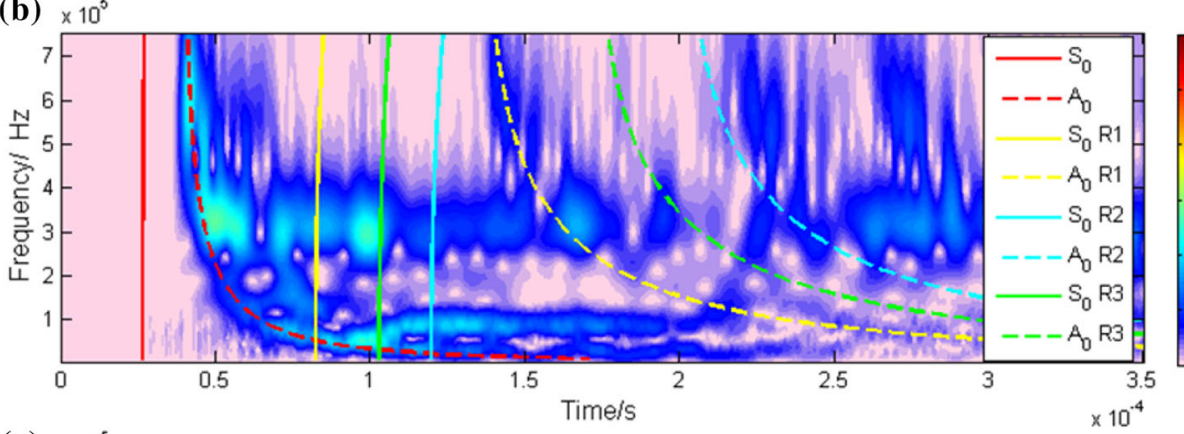

$-\int_{-3}^{x^{6}} \cdot 0^{-3}$
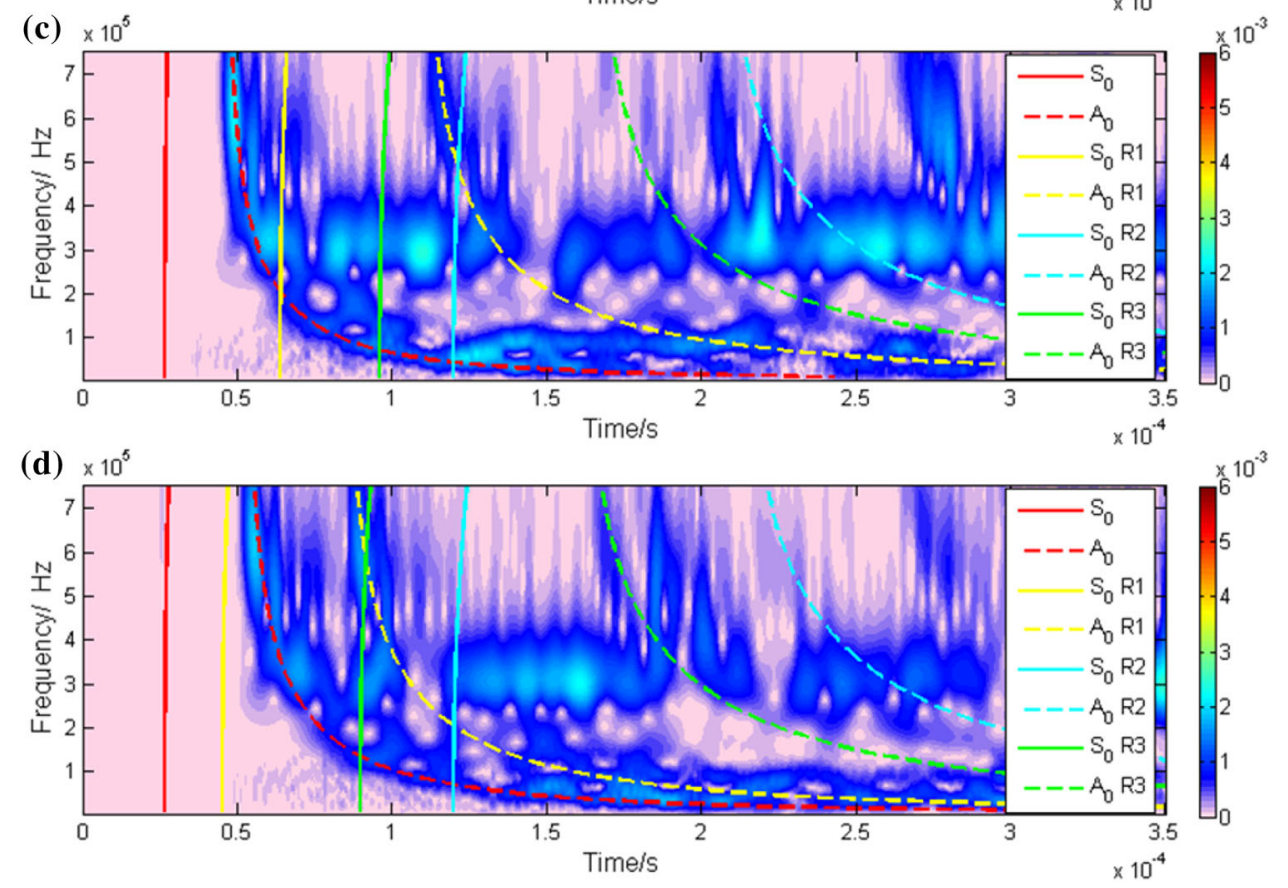

mately 50, 210 and $320 \mathrm{kHz}$, with the peak frequencies being in the $210 \mathrm{kHz}$ region. The un-bonded double-layer specimen also features significant content under $100 \mathrm{kHz}$, though does not feature a peak at $210 \mathrm{kHz}$ but has a single prominent peak at around $320 \mathrm{kHz}$. Low-amplitude content in the 400-600 $\mathrm{kHz}$ region is also visible. The bonded specimen features a narrow peak centred at around $50 \mathrm{kHz}$ with minimal content visible across the rest of the spectrum. As the source remains constant across all specimens, the change in peak frequencies between specimens is because of the bond condition of the specimen that the waves are propagating through and not the nature of the source. This factor must therefore be considered if a peak-frequency-based analysis method is to be utilised to differentiate between failure mechanisms, as the propagation path will affect the mechanism detected.

From the PSD plots, there is no significant change in peak frequency resulting from variation in propagation distance. To provide greater comparison of the overall spectral content, rather than just the peaks, partial-power characteristics have been investigated, with the percentage of total signal 
Fig. 8 Example WT coefficient plots and modified group-velocity curves for an out-of-plane source applied to the adhesively-bonded specimen with source-sensor propagation distances of a $50 \mathrm{~mm}, \mathbf{b} 100$ $\mathrm{mm}, \mathbf{c} 150 \mathrm{~mm}$ and $\mathbf{d} 200 \mathrm{~mm}$
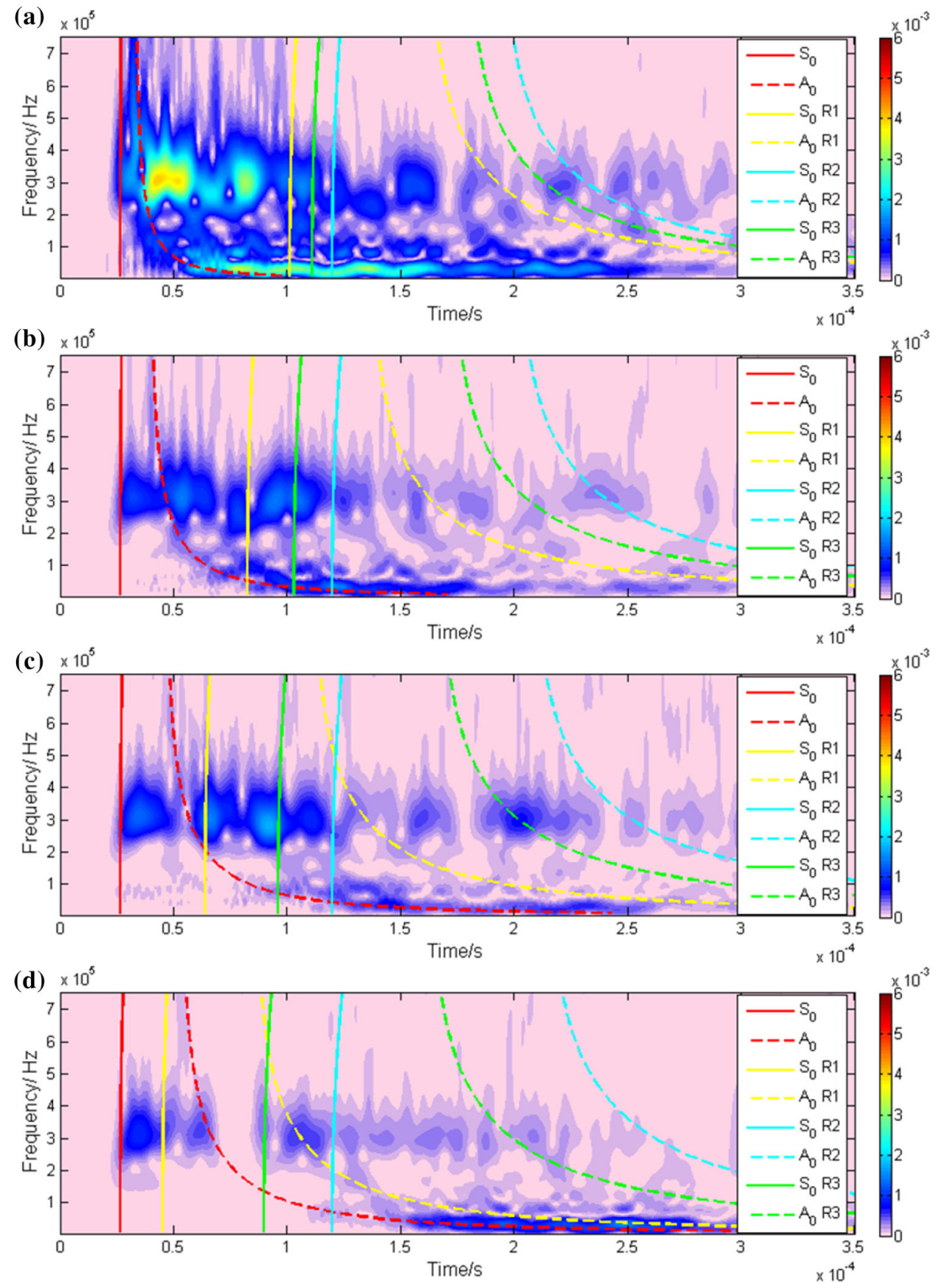

energy contained in each of the four main frequency bands identified being calculated. The selected frequency bands are $0-100 \mathrm{kHz}, 100-250 \mathrm{kHz}, 250-375 \mathrm{kHz}$ and $>375 \mathrm{kHz}$. Signal energy was calculated as the integral of the square of the signal over the entire record: $E=\int_{0}^{t} V^{2}(t) d t$ [22]. Figure 11 illustrates the proportion of energy contained within each frequency band and its variation with propagation distance. As in the PSD plots, there is a clear difference between specimens, with the highest percentages of energy being contained in the bands covering the peak frequencies previously discussed. In this case however, variation with propagation distance can also be identified. In the single and un-bonded double-layer specimens, the variation is minimal and increasing or decreasing trends are inconsistent, apart from a slight decrease in the low-frequency band. The bonded specimen, however, exhibits a significant and consistent increase in low-frequency content and decrease in all higher-frequency content with increasing propagation distance. It can therefore be seen that while the peak frequencies remain consistent across the source-sensor distances tested, the spectral content does experience a significant change 
Fig. 9 WT coefficient plot from an out-of-plane source applied to the adhesively-bonded specimen with a propagation distance of $200 \mathrm{~mm}$ and modified group-velocity curves for $1 \mathrm{~mm}$ (representing the adherend thickness) and $2.2 \mathrm{~mm}$ (representing entire specimen thickness) aluminium sheets

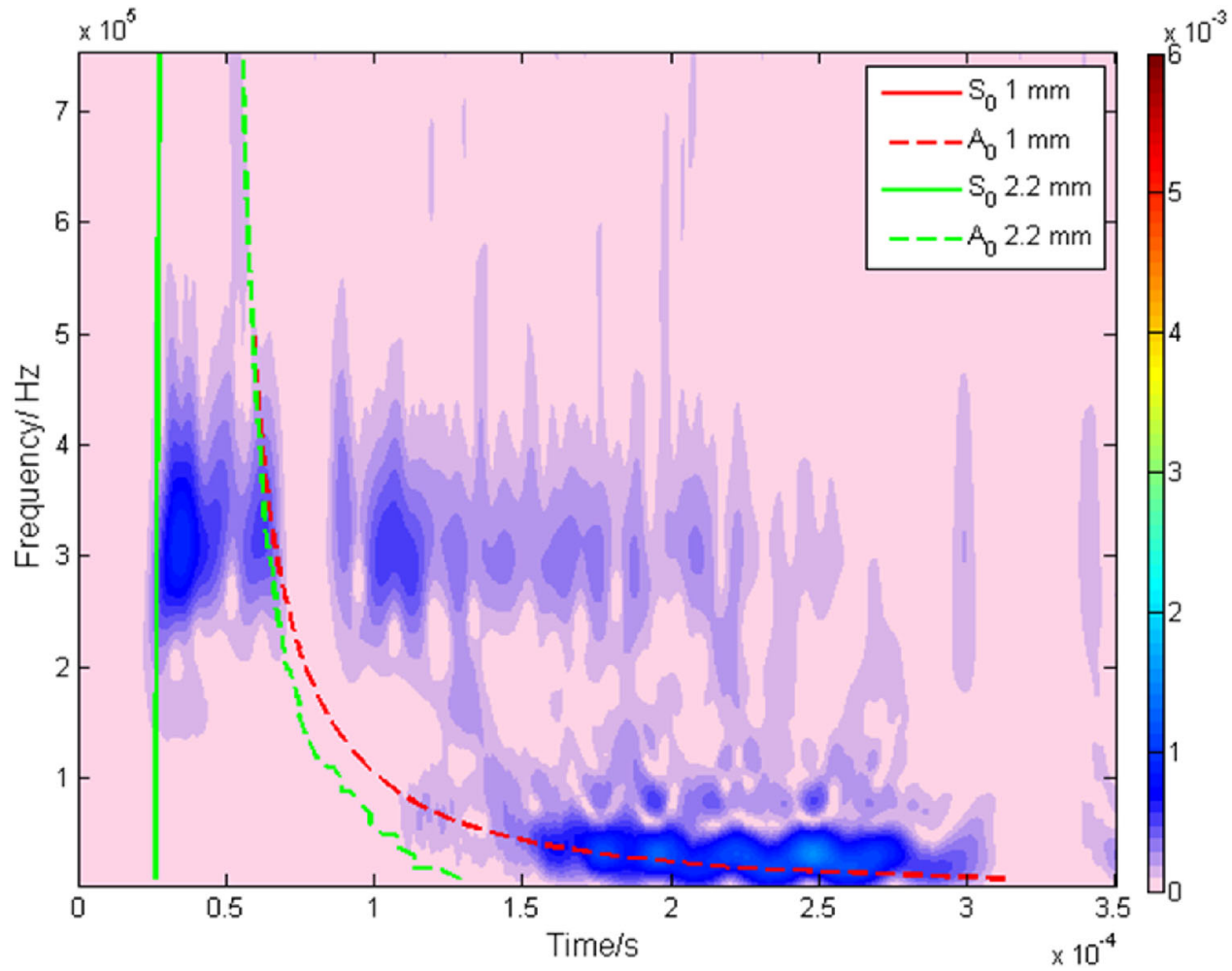

in the bonded specimen as the adhesive attenuates highfrequency components of the signal.

While the two previously-described methods provide insight into the variation in spectral content regarding sourcesensor distance, it should be remembered that most of the signal analysed comprises of edge reflections, each of which has a different propagation distance, and therefore potentially different spectral content, from the initial wave. It can be seen from the WT coefficient plots for the bonded specimen (Fig. 8 ) that, even at the shortest source-sensor distance, the edge reflections contain very little high-frequency content due to the attenuation over their additional propagation distance. As the changes in source-sensor distance are minimal, compared to the propagation distances of the reflections, the effects of varying source-sensor distance are somewhat masked when the entire signal is analysed. Complete isolation of the initial wave in either time or time-frequency domain is limited by the overlapping of edge reflections with low-velocity components of the initial wave, as can be seen in the WT coefficient plots. Therefore, to demonstrate the effect of propagation distance on the spectral content of the initial wave, a similar approach to that taken by Zhang et al. [15] has been utilised, and the peak WT coefficient in the low- $(<100 \mathrm{kHz})$ and mid- (200-400 kHz) frequency regions corresponding to the arrival of the initial $\mathrm{A}_{0}$ wave have been extracted. The ratio between these peak WT coefficients has then been used to define the changes with propagation distance and between specimens. This ratio is defined below as Eq. (3):
Ratio $=\frac{W T \text { Peak } k_{\text {low freq. }}}{W T \text { Peak }}$

Figure 12 illustrates the change in this ratio of low- to mid- frequency content with increasing propagation distance for the three specimens tested. The single-sheet specimen exhibits a very minor decrease in ratio, implying that the low-frequency component becomes less prominent over distance. There is slight variation in ratio for the un-bonded double-layer, though there is no distinguishable increasing or decreasing trend, the low-frequency peak value is consistently slightly higher than the mid-frequency peak value. The bonded specimen features a similar ratio to the unbonded specimen at a propagation distance of only $50 \mathrm{~mm}$, though increases exponentially with increasing propagation distance, indicating that within the initial wave the midto high-frequency components are being attenuated significantly more than low-frequency components as the wave propagates.

In summary, the three methods of frequency analysis utilised indicate significant variation in spectral content between the single, un-bonded and bonded specimens despite use of the same source. The presence of an additional aluminium sheet causes a slight increase in the high-frequency content recorded, while the presence of an adhesive layer causes attenuation of mid- to high-frequency components resulting in a dramatic drop in peak frequency. The effect of propagation distance is seen to have minimal effect on the fre- 
(I) Single-sheet

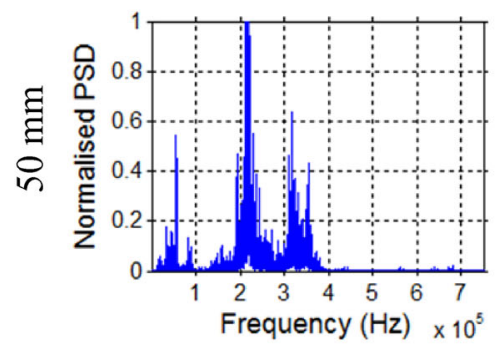

(a)

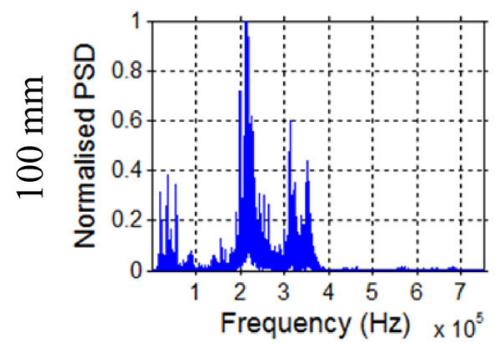

(b)

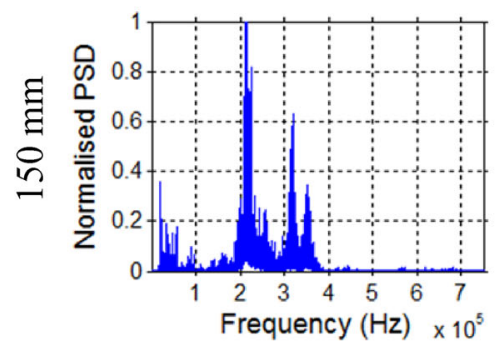

(c)

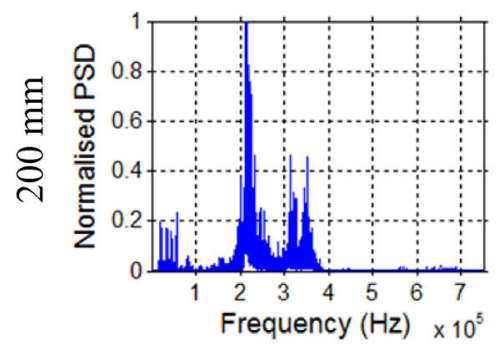

(d)
(II) Un-bonded double-layer

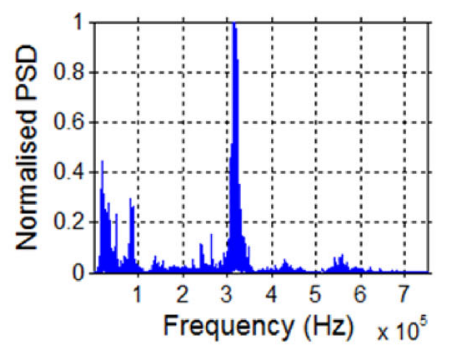

(e)

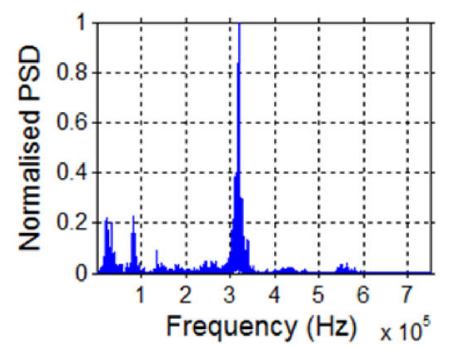

(f)

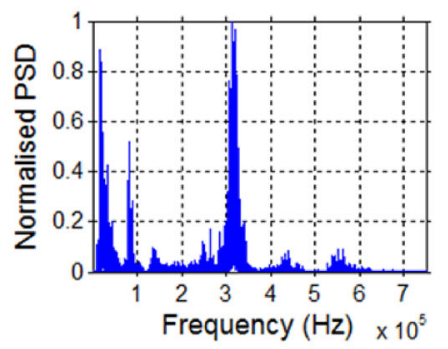

(g)

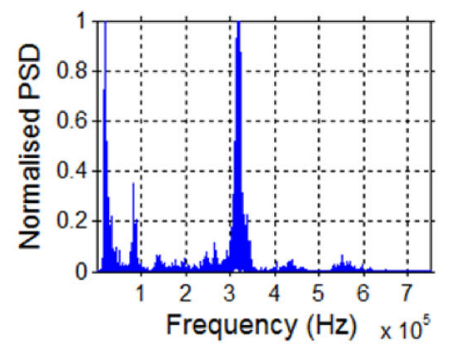

(h)
(III) Adhesively-bonded

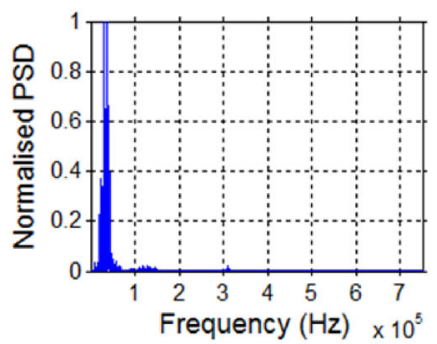

(i)

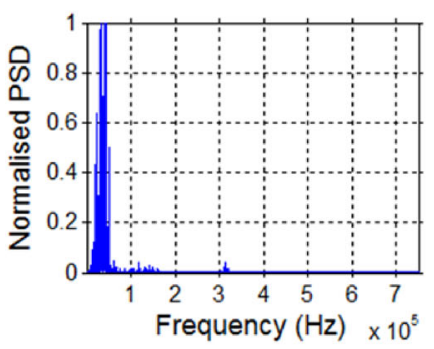

(j)

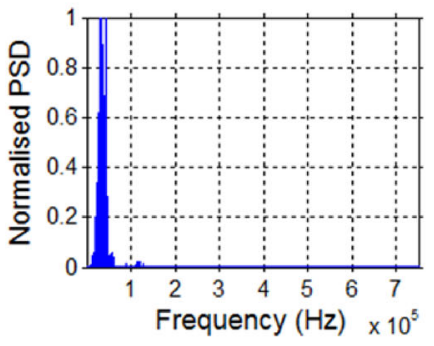

(k)

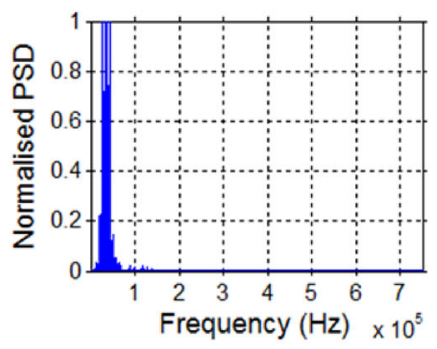

(I)
Fig. 10 Normalised Power Spectral Density plots indicating spectral content of the entire recorded signals for an out-of-plane source on a single-sheet specimen (1st column), an un-bonded double-layer spec-

quency content of the single and un-bonded specimens, while the attenuation introduced by the adhesive results in a significant variation in spectral content with varying propagation distance in the bonded specimen. The increased volume of the bonded specimen should result in an overall increase in geometric attenuation across all frequencies, due to spreading of the wavefront, while the selective attenuation of the mid- to high-frequency content is believed to be due to the viscoelastic nature of the adhesive. Although the frequency ranges imen (no adhesive) (2nd column) and an adhesively-bonded specimen (3rd column), at propagation distances of $50 \mathrm{~mm}$ (1st row), $100 \mathrm{~mm}$ (2nd row), $150 \mathrm{~mm}$ (3rd row) and $200 \mathrm{~mm}$ (4th row)

under consideration are different, these findings appear to be in good agreement with those of Heller et al. [16], who reported a loss of the higher order wave modes, which exist at high-frequency, in the presence of an adhesive layer.

In AE testing of bonded joints these factors should be considered when selecting sensor placement, as the bond status of the propagation path to the sensor will affect the received spectral content. For example, in a double-cantilever-beam test or similar, the source (the crack front) will be in the cen- 
(a)

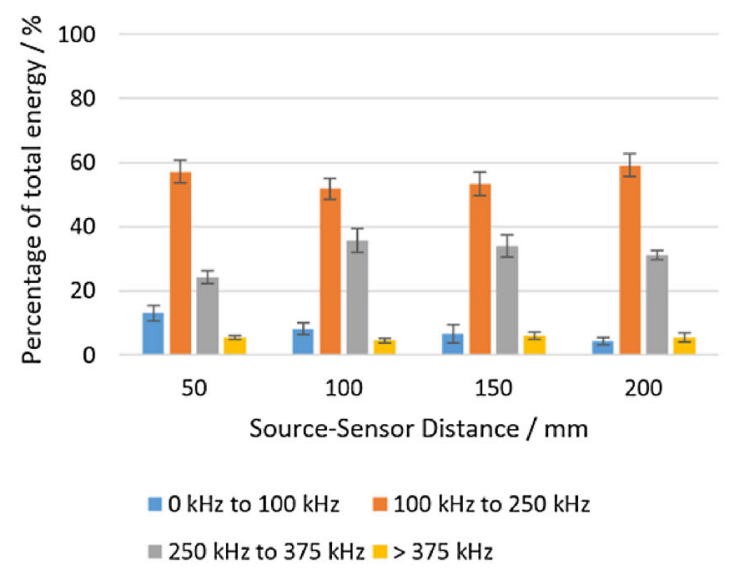

(b)

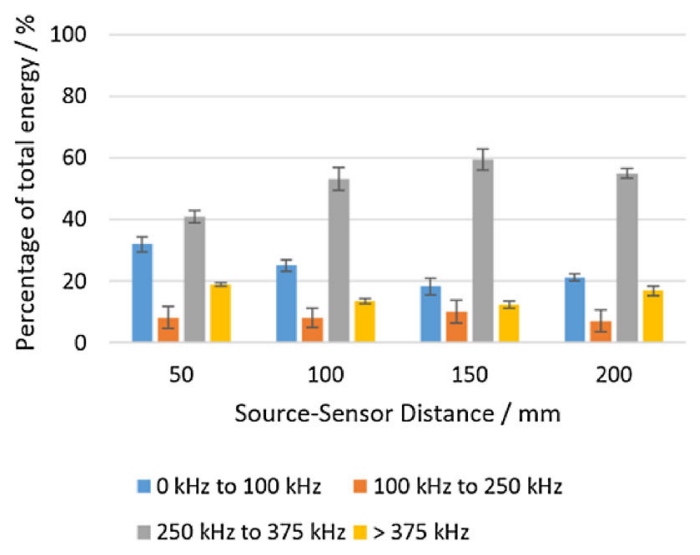

(c)

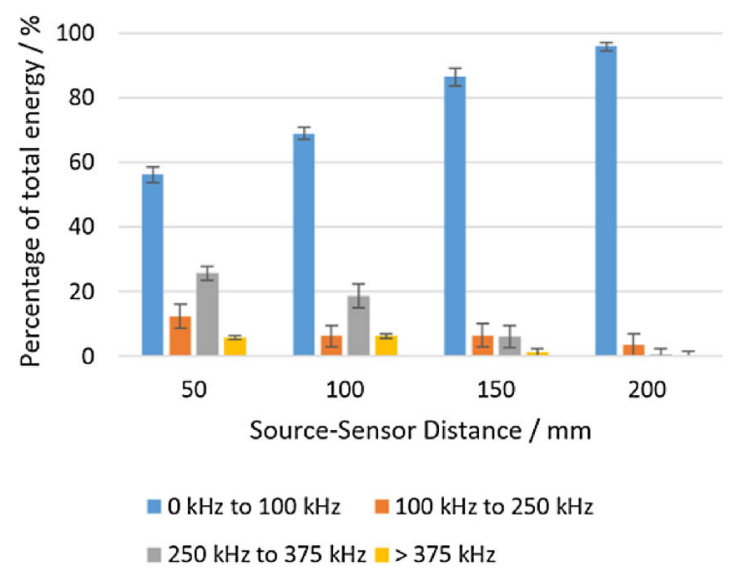

Fig. 11 Mean percentage of AE energy in key frequency bands at varying source-sensor distances for; a single sheet specimen, b un-bonded double-layer specimen (no adhesive) and $\mathbf{c}$ adhesively-bonded specimen (Error bars show standard deviation)

tral region of the specimen, with an un-bonded section at the opening end and a fully bonded section at the opposite end. Placement of the sensor at the opening end will result in minimal attenuation from source to sensor as the waves propagate through a single adherend, while placement of the sensor at the closed end will result in attenuation of highfrequency components as the waves propagate through the bonded region. In small specimens typical of most previous studies, where edge reflections are prominent, the effect of this may be negligible due to the reflections propagating back and forth across the entire specimen. In large specimens however, where edge reflections are less significant, the sensor location may have a much greater effect on the recorded spectral content.

\subsection{Effects of Source Orientation}

The resultant WT coefficient plots from application of an inplane source on the edge of the specimens (as shown in Fig. 2)

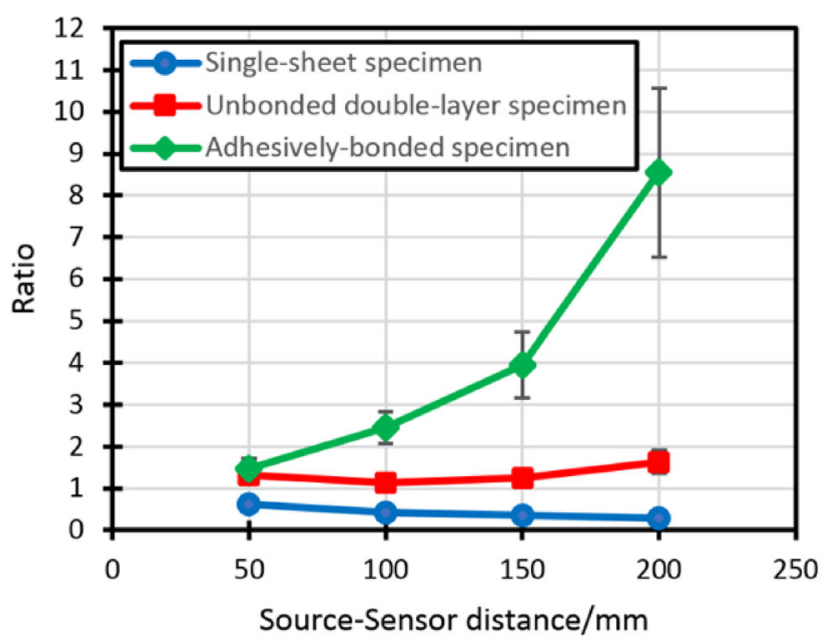

Fig. 12 Mean WT coefficient peak ratio between low $(<100 \mathrm{kHz})$ and mid (200-400 kHz) regions of initial $A_{0}$ wave with increasing source-sensor distance for the single-sheet, un-bonded double-layer (no adhesive) and adhesively-bonded specimens. Error bars show standard deviation 
Fig. 13 Example WT coefficient plots and modified group-velocity curves for an in-plane source applied at a source-sensor distance of 250 $\mathrm{mm}$ to the edge of $\mathbf{a}$ the single-sheet specimen, $\mathbf{b}$ the un-bonded double-layer specimen, $\mathbf{c}$ the upper adherend of the adhesively-bonded specimen and $\mathbf{d}$ the adhesive layer of the adhesively-bonded specimen
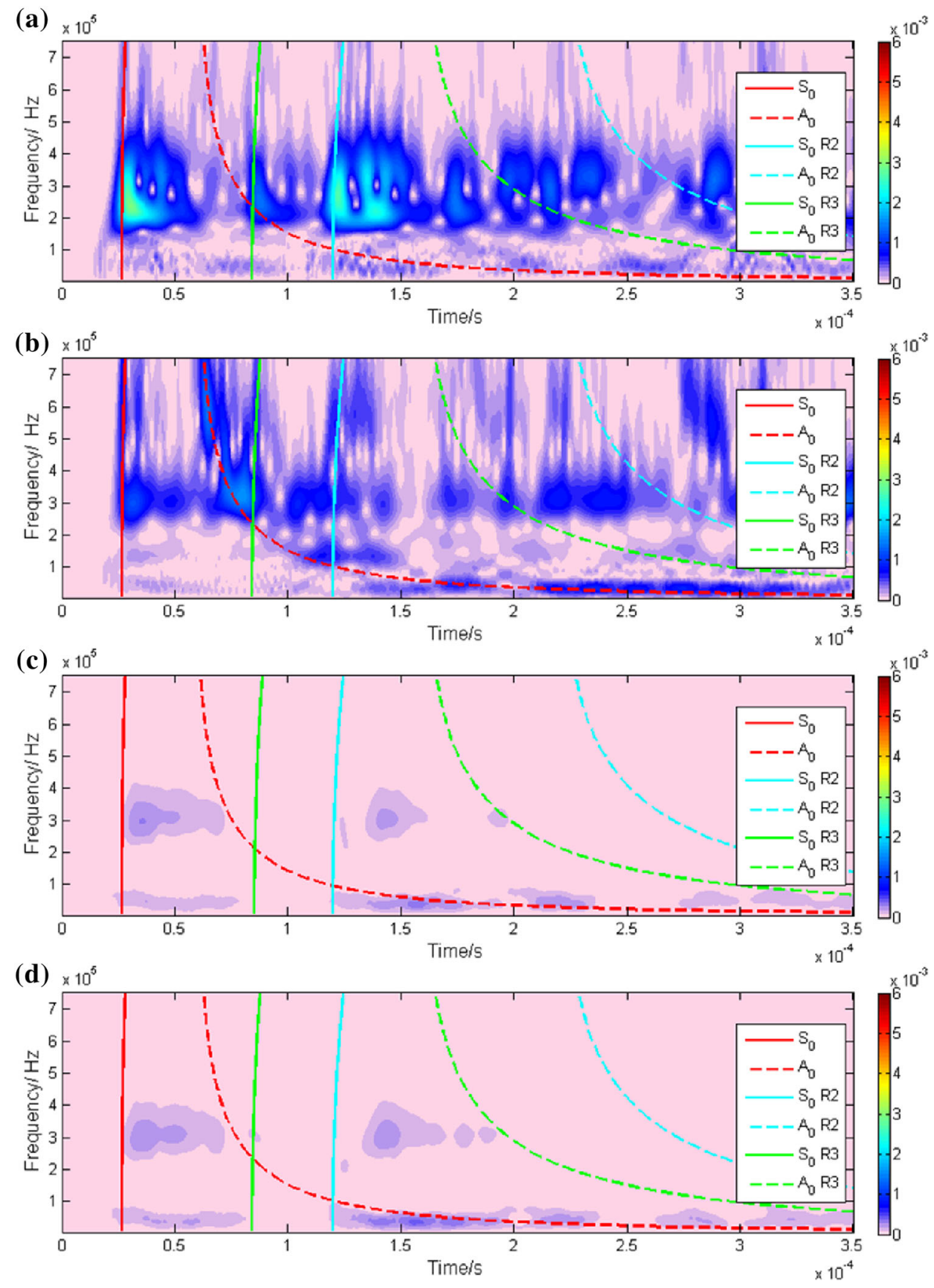

are shown in Fig. 13. These differ greatly from those acquired with an out-of-plane source as the $\mathrm{S}_{0}$ mode becomes more significant. In the single-sheet specimen, the high-energy regions all exist within the $200-400 \mathrm{kHz}$ region and are seen to correspond to the $\mathrm{S}_{0}$ mode and its multiple reflections. There is little content which can be positively identified as corresponding to the $\mathrm{A}_{0}$ mode. As in the tests using an out-ofplane source, the un-bonded double-layer specimen exhibits much greater high-frequency content above $400 \mathrm{kHz}$. The $\mathrm{S}_{0}$ mode, and subsequent reflections, can be identified as occur- ring across the frequency range of approximately 200-750 $\mathrm{kHz}$. Unlike in the single-sheet specimen, the initial $\mathrm{A}_{0}$ wave can also be clearly identified, although its reflections are less clearly defined. Compared to the single sheet, the un-bonded double-layer specimen also exhibits a much higher amplitude low-frequency component corresponding to the $\mathrm{A}_{0}$ mode.

For both source depths tested, the bonded specimen exhibits a clear initial $\mathrm{S}_{0}$ wave, present across the midfrequency band of $200-400 \mathrm{kHz}$, the reflections of this mode however are not as clearly defined as in the other speci- 
mens. As was found in the previously discussed tests, the adhesively-bonded specimen appears to quickly attenuate any high-frequency content. This results in there being no significant content above $400 \mathrm{kHz}$ and the reflections present in the other specimens being attenuated significantly before arrival at the sensor. The peak WT coefficient occurs in the low-frequency region below $100 \mathrm{kHz}$, which appears to correspond approximately to the $\mathrm{A}_{0}$ mode. It is however noted that the arrival of this low-frequency component is slightly earlier than predicted by the group-velocity curves. Overall, both source depths tested on the bonded specimen provide very similar results.

In general, the in-plane source results in a significant increase in the proportion of energy propagating in the $\mathrm{S}_{0}$ mode, when compared to an out-of-plane source. This result is to be expected, based on the previous demonstrations of this characteristic such as those by Gorman [23] and Hamstad et al. [12]. From these studies, it is also to be expected that sources located at the mid-depth of the specimen will excite the purest $\mathrm{S}_{0}$ mode, while offset from the mid-depth will introduce a greater flexural $\mathrm{A}_{0}$ component. The source was applied at the mid-depth of the single-sheet specimen as accurately as was possible for the source type used. The resulting WT coefficient plots are seen to exhibit a clear $\mathrm{S}_{0}$ wave and its subsequent reflections, with little clear evidence of any significant $\mathrm{A}_{0}$ mode, providing a perfect example of the expected behaviour. The un-bonded double-layer specimen, on which the source was applied at the mid-depth of the upper adherend, shows a greatly increased $\mathrm{S}_{0}$ component but still exhibits a well-defined $\mathrm{A}_{0}$ mode. In the bonded specimen, it was expected that there may be an observable difference between the two source locations; based on the finding that individual Lamb waves are propagating in each layer, the source applied to the mid-plane of the adherend should create the purest $\mathrm{S}_{0}$ mode, while the source applied to the adhesive is offset from the plane in which the recorded waves are propagating, and should thus create an increased $\mathrm{A}_{0}$ component.

Applying the previously used method of creating a ratio between peak WT coefficients gives a clear differentiation between the specimens. In this case the peaks were taken from the mid-frequency $(200-400 \mathrm{kHz})$ region of the $S_{0}$ wave and the low-frequency $(<100 \mathrm{kHz})$ region of the $\mathrm{A}_{0}$ wave. The ratio between the peaks is calculated as per Eq. (4) below:

Ratio $=\frac{W T \text { Peak }}{A_{0} \text { low freq. }}$ WT Peak $S_{S_{0} \text { mid freq. }}$

The resulting ratios, presented in Fig. 14, show the single sheet provides the lowest ratio, with a mean of 0.2 , indicating that the $\mathrm{S}_{0}$ mode is clearly dominant. The low-frequency $\mathrm{A}_{0}$ peak is however still the highest in the un-bonded doublelayer specimen with a ratio of 1.4. Both source depths on the

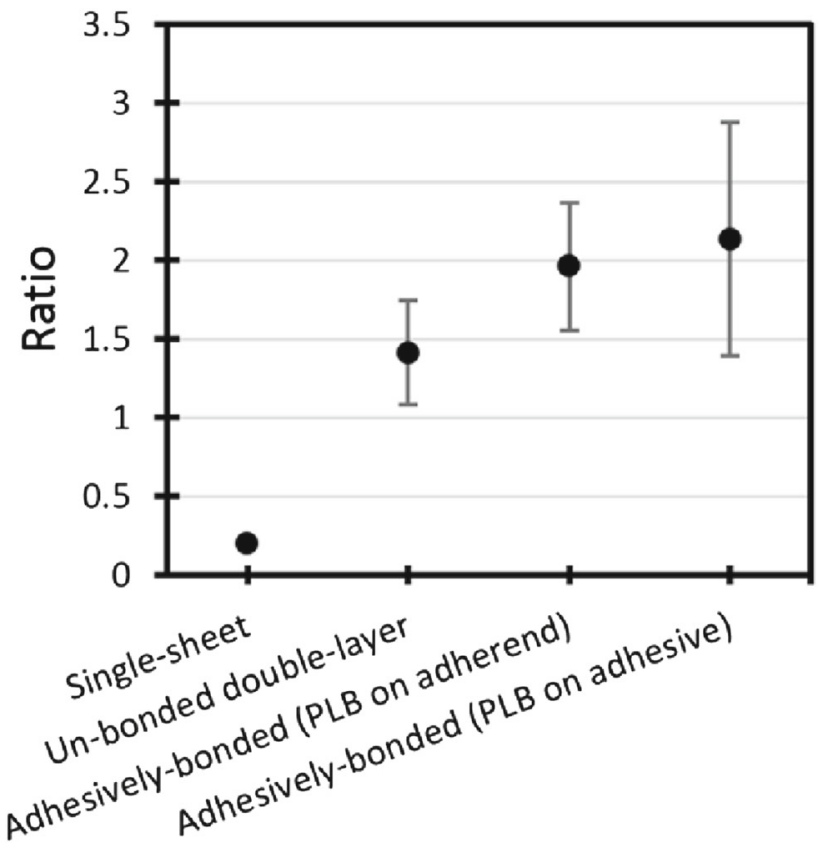

Fig. 14 Mean WT coefficient peak ratios (Peak $A_{0} /$ Peak $S_{0}$ ) for an inplane source located $250 \mathrm{~mm}$ from the sensor. Error bars show standard deviation

bonded specimen yield similar results showing dominance of the low-frequency $\mathrm{A}_{0}$ component, with ratios of 1.9 and 2.1 for the source applied to the adherend and adhesive respectively. The difference between the two source depths cannot however be considered significant due to the variation in ratio within each of these tests.

The minimal difference between source locations may be in part due to the low total thickness of the specimens, resulting in minimal offset from the central plane regardless of source location on the edge. For comparison, the plates considered by Hamstad et al. [12] were $4.7 \mathrm{~mm}$ thick and a maximum offset from the central plane of $1.88 \mathrm{~mm}$ was considered. In this case the offset from the centre of the adhesive was only $0.6 \mathrm{~mm}$. While the change in ratio between $\mathrm{A}_{0}$ and $\mathrm{S}_{0}$ peaks can be largely attributed to the wave modes excited in the specimens, the attenuation of high-frequency content in the adhesively-bonded specimen will also contribute towards the relative dominance of the low-frequency $\mathrm{A}_{0}$ component, by attenuation of the mid- to high-frequency $\mathrm{S}_{0}$ waves.

Ultimately, as was seen for an out-of-plane source, the addition of a second adherend and of an adhesive layer does not change the effective wave propagation modes from a simulated in-plane source, with the recorded waveforms showing good correspondence to the theoretical dispersion curves of a single adherend. The adhesive layer is again seen to introduce greater attenuation, particularly of higher frequency components, and most noticeably in reflections where the propagation distance is greatest. 


\section{(I) Sensor Side}

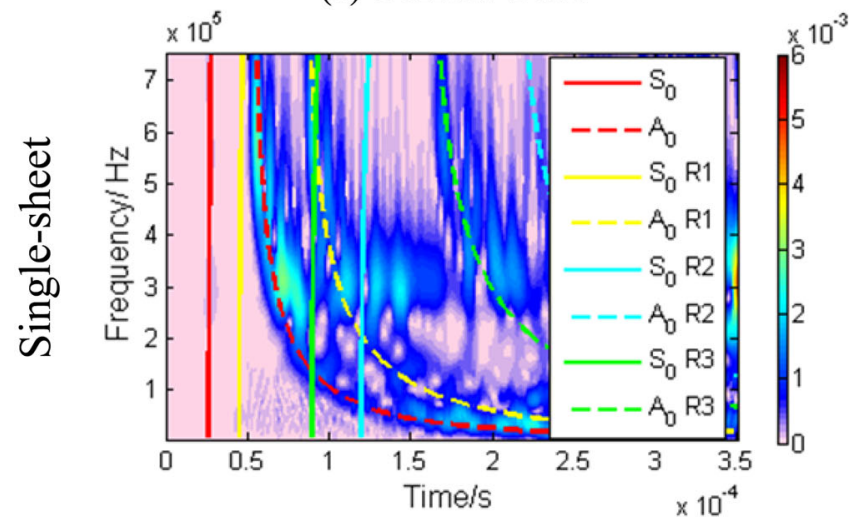

(a)

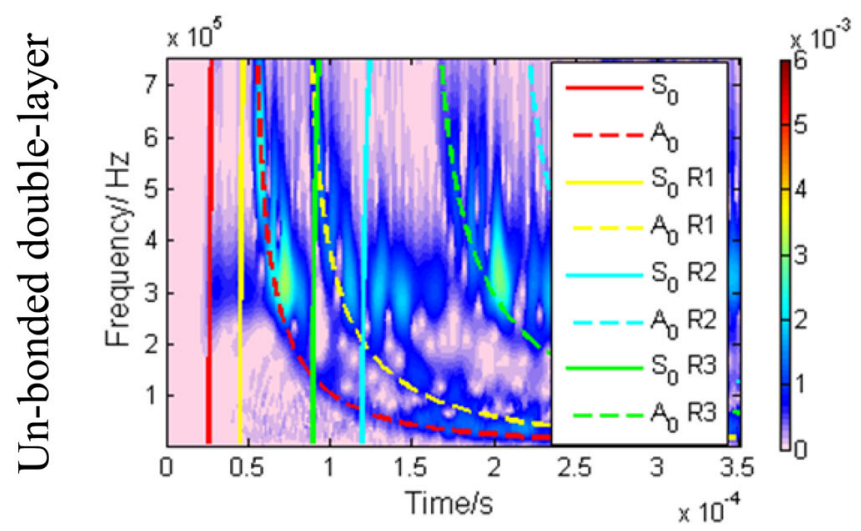

(c)

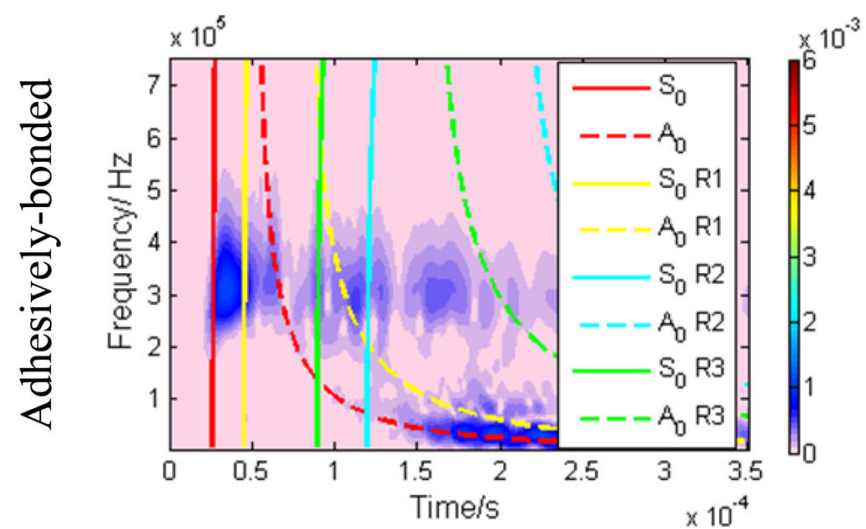

(e)

Fig. 15 The effect of source side, with respect to the sensor, for: A single-sheet specimen (1st row), an un-bonded double-layer specimen (no adhesive) (2nd row) and an adhesively-bonded specimen (3rd row). The 1st column shows WT plots for sources applied on the same face as

\subsection{Effects of Source Side}

The tests were completed initially with the source and sensor on the same side of the specimen, and were then repeated
(II) Opposite Side

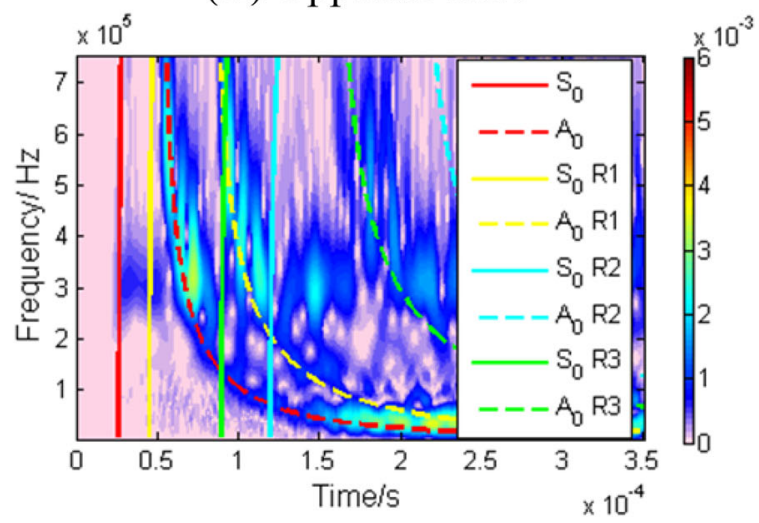

(b)

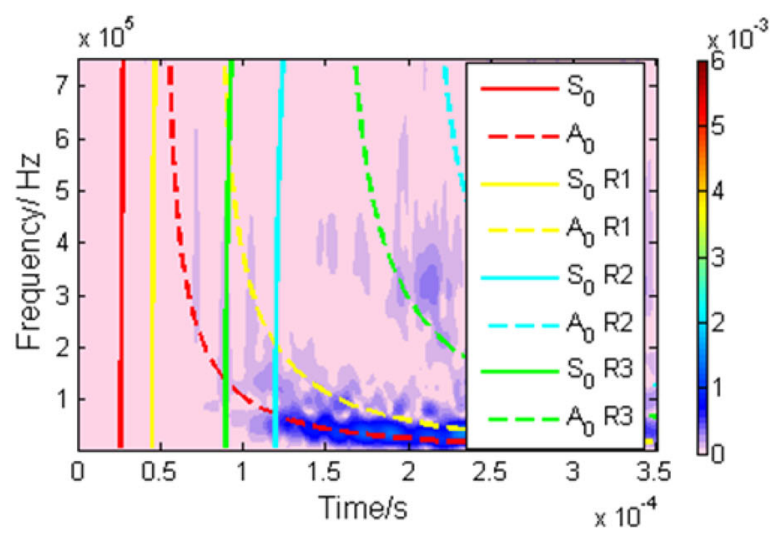

(d)

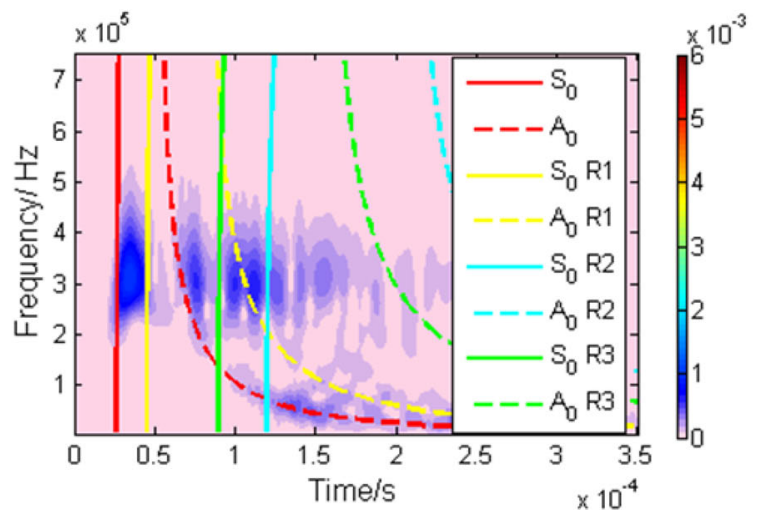

(f)

the sensor, the 2nd column shows WT plots for sources applied on the opposite face to the sensor. Example results shown from tests conducted at source-sensor distance of $200 \mathrm{~mm}$

with the source applied on the opposite surface to the sensor, to identify the effect of transmission through the specimens. As seen in Fig. 15, throughout all specimens it was found that, as would be expected, the arrival times of the various 
wave modes and their reflections remained unchanged by this change in source position. In the single-sheet specimen, the side that the source is applied to has little significant effect on the signal recorded. In the un-bonded double-layer however, there is a vast difference. Application of the source on the opposite side to the sensor results in only the low frequency components $(<100 \mathrm{kHz}$ ) being transferred at any significant amplitude. The higher frequency components, which are dominant when the source and sensor are located on the same side, appear not to transfer across the interface between the source-side sheet and sensor-side sheet. This results in significantly reduced signal amplitude and energy, as well as a change in spectral content. Investigation of the bonded specimen reveals negligible change in the signal resulting from changing the side of the source. It could be expected that signals arriving from a source applied to the opposite side of the specimen from the sensor, should be subject to a higher level of attenuation than those on the same side, due to the greater propagation distance and the effects of propagation through the adhesive layer. Analysis of AE energy recorded from each source location, however, showed no significant difference between sides. This is assumed to be due to any difference occurring due to transmission through the adhesive layer being negligible compared to the other factors affecting the recorded signal. It is expected that in the case of thicker adherends, or a thicker adhesive layer, that the difference would become significant. Frequency analysis also revealed negligible difference between the sides to which the source was applied.

\section{Conclusions}

This research presents, for the first time, a systematic investigation of $\mathrm{AE}$ wave propagation in metal-to-metal adhesively-bonded joints. Wave propagation has been investigated in three specimens; a single aluminium sheet, two aluminium sheets placed together without adhesive and an adhesively-bonded specimen, using both in-plane and out-ofplane sources applied at varying distances from the sensor. Analysis in the time-frequency domain by use of wavelet transforms with overlaid dispersion curves, and in the frequency domain by use of FFT and partial power techniques, allows the following general conclusions to be made:

a. The use of wavelet transforms with modified dispersion curves allows positive identification of reflections corresponding to each edge of the specimens, as well as Lamb modes contained in the initial wave, while using only a single transducer and simple pencil lead break source.

b. It has been demonstrated that the wave modes propagating in all specimens tested can be suitably approximated by the Lamb modes of a single adherend, and are largely insensitive to bond status. Standard RayleighLamb equations may therefore be used to calculate wave velocities in bonded specimens regardless of bond quality, bond thickness or specification of the secondary adherend.

c. The presence of additional layers in the specimen results in significant changes in peak frequency recorded from the same source. Care must therefore be taken in the use of peak-frequency-based methods of AE source characterisation, as the bond status of the wave propagation path may lead to erroneous identification of failure mechanisms.

d. The viscoelastic nature of the adhesive results in attenuation of high-frequency content in the adhesively-bonded specimen, leading to spectral content varying with propagation distance. This may be critical in AE testing of large specimens as similar sources at different locations will produce vastly different signals, again potentially leading to erroneous identification of failure mechanisms.

e. The side of the specimen to which the AE source is applied, relative to the sensor, is seen to have negligible effect on the signals recorded for both the single-sheet, and the bonded specimens which were tested. The lack of bonding in the un-bonded double-layer specimen, results in loss of the dominant high-frequency components when the source is moved from the sensor-side of the specimen to the opposite side. This change in spectral content is accompanied by a corresponding drop in signal amplitude and energy, as the high frequency components do not transfer across the interface from one layer to the other.

Work conducted on AE monitoring of adhesively-bonded joints so far has focused on relatively small test specimens, in which wave propagation has been of little concern. As this work moves towards full-scale testing and industrial application, however, the features highlighted in this paper will begin to play a critical part in accurate detection and characterisation of failure mechanisms of various adhesively-bonded joints by acoustic emission.

Acknowledgements The research did not receive any specific grant from funding agencies in the public, commercial, or not-for-profit sectors.

Open Access This article is distributed under the terms of the Creative Commons Attribution 4.0 International License (http://creativecomm ons.org/licenses/by/4.0/), which permits unrestricted use, distribution, and reproduction in any medium, provided you give appropriate credit to the original author(s) and the source, provide a link to the Creative Commons license, and indicate if changes were made. 


\section{References}

1. Wong, B.S.: Non-destructive evaluation of composites: detecting delamination defects using mechanical impedance, ultrasonic and infrared thermographic techniques. In: Karbhari, V.M. (ed.) Nondestructive Evaluation (NDE) of Polymer Matrix Composites, pp. 279-305. Woodhead Publishing Limited, Singapore (2013)

2. Adams, R.D.: Nondestructive testing. In: da Silva, L.F.M. (ed.) Handbook of Adhesion Technology, pp. 1049-1069. Springer, Bristol (2011)

3. Bak, M.K., Kalaichelvan, K.: Evaluation of failure modes of pure resin and single layer of adhesively bonded lap joints using acoustic emission data. Trans. Indian Inst. Metals 68, 73-82 (2015)

4. Croccolo, D., Cuppini, R.: A methodology to estimate the adhesive bonding defects and the final releasing moments in conical joints based on the acoustic emissions technique. Int. J. Adhes. Adhes. 26, 490-497 (2006)

5. Curtis, G.J.: Acoustic emission energy relates to bond strength. Non-destr. Test. 33, 249-257 (1975)

6. Droubi, M.G., Stuart, A., Mowat, J., Noble, C., Prathuru, A.K., Faisal, N.H.: Acoustic emission method to study fracture (Mode-I, II) and residual strength characteristics in composite-to-metal and metal-to-metal adhesively bonded joints. J. Adhes. 94(5), 347-386 (2017)

7. Ducept, F., Davie, P., Gamby, D.: Mixed mode failure criteria for a glass/epoxy composite and an adhesively bonded composite/composite joint. Int. J. Adhes. Adhes. 20(3), 233-244 (2000)

8. Dzenis, A., Saunders, I.: On the possibility of discrimination of mixed mode fatigue fracture mechanisms in adhesive composite joints by advanced acoustic emission analysis. Int. J. Fract. 117, 23-28 (2002)

9. Galy, J., Moysan, J., El Mahi, A., Ylla, N., Massacret, N.: Controlled reduced-strength epoxy-aluminium joints validated by ultrasonic and mechanical measurements. Int. J. Adhes. Adhes. 72, 139-146 (2016)

10. Senthil, K., Arockiarajan, A., Palaninathan, R.: Experimental determination of fracture toughness for adhesively bonded composite joints. Eng. Fract. Mech. 154, 24-42 (2016)

11. Pashmforoush, F., Khamedi, R., Fotouhi, M., Hajikhani, M., Ahmadi, M.: Damage classification of sandwich composites using acoustic emission technique and k-means genetic algorithm. J. Nondestr. Eval. 33, 481-492 (2014)
12. Hamstad, M.A., O’Gallagher, A., Gary, J.: A wavelet transform applied to acoustic emission signals: part 1: source identification. J. Acoust. Emiss. 20, 39-61 (2002)

13. Hamstad, M.A., O'Gallagher, A., Gary, J.: A wavelet transform applied to acoustic emission signals: part 2: source location. J. Acoust. Emiss. 20, 62-82 (2002)

14. Hamstad, M.A.: Comparison of wavelet transform and ChoiWilliams distribution to determine group velocities for different acoustic emission sensors. J. Acoust. Emiss. 56, 40-59 (2008)

15. Zhang, X., Feng, N., Wang, Y., Shen, Y.: An analysis of the simulated acoustic emission sources with different propagation distances, types and depths for rail defect detection. Appl. Acoust. 86, 80-88 (2014)

16. Heller, K., Jacobs, L.J., Qu, J.: Characterization of adhesive bond properties using lamb waves. NDT \& E Int. 33, 555-563 (2000)

17. ASTM.: E 976-99 Standard Guide for Determining the Reproducibility of Acoustic Emission Sensor Response. ASTM (1999)

18. Engelberg, S.: Understanding sampling. In: Engelberg, S. (ed.) Digital Signal Processing An Experimental Approach, pp. 3-15. Springer, Jerusalem (2008)

19. Suzuki, H., Kinjo, T., Hayashi, Y., Takemoto, M., Ono, K.: Wavelet transform of acoustic emission signals. J. Acoust. Emiss. 14(2), 69-84 (1996)

20. Su, Z., Ye, L.: Fundamentals and analysis of lamb waves. In: Su, Z., Ye, L. (eds.) Identification of Damage Using Lamb Waves-From Fundamentals to Applications, pp. 15-53. Springer, Berlin (2009)

21. Siefried, R., Jacobs, L.J., Qu, J.: Propagation of guided waves in adhesive bonded components. NDT \& E Int. 35, 317-328 (2002)

22. Droubi, M.G., Reuben, R.L., White, G.: Statistical distribution models for monitoring acoustic emission (AE) energy of abrasive particle impacts on carbon steel. Mech. Syst. Signal Process. 30, 356-372 (2012)

23. Gorman, M.R.: AE source orientation by plate wave analysis. J. Acoust. Emiss. 9(4), 283-288 (1991) 